\title{
Die Entstehung des Blutes und der ersten Gefässe im Hühnerei.
}

\author{
Von \\ Dr. J. Disse, \\ I. Assistenten am anatomischen Institut zu Strassburg. \\ Hierzu Tafel XXVI, XXVII, XXVIII.
}

So wenig wie die Ansichten über die Genese des mittleren Keimblattes beim Vogelei auch nur in den hauptsächlichsten Puncten in Uebereinstimmung zu bringen sind, so wenig herrscht Klarheit uiber die Beantwortung der Frage nach der ersten Anlage des Blutes und der Blutbahnen.

Musste schon ein Beobachter vom Range Remak's sich ausser Stande erklären, genaner anzugeben, wie die Blutzellen entstehen: konnten schon die nächsten Nachfolger ihre Beobachtungen nicht in übereinstimmender Weise deuten: dariiber waren sie wenigstens mit Remak einig, dass das Blut im mittleren Keimblatt entstände, und ebenso von Furchungszellen abstamme, sowie die übrigen Producte des Mesoblasten. So weit wenigstens wahren Afanasieff und Klein den principiellen Standpunct Remak's; schon früher hatte Kölliker viel enger an Remak sich angeschlossen. Einer ganz verschiedenen Auffassung suchte dagegen His Geltung zu verschaffen. Er glaubt dem Blute, sowie den Bindesubstanzen tiberhaupt einen gesonderten Ursprung zuschreiben zu muissen; sie sollen nicht von Zellen, die aus dem Bildungsdotter stammen, abzuleiten sein, sondern von den Elementen des Nahrungsdotters, denen man bisher plastische Eigenschaften nicht hatte zuerkennen wollen. 
Es soll sich nach $H$ is der Wirbelthierleib aufbauen aus zwei getrennten Keimen. Der eine, „Archiblast", dessen Material der sich furchende Bildungsdotter liefert, ist die Quelle der physiologisch bedeutsamen, den Functionen des animalen Lebens vorstehenden Gewebe, nämlich der Epithelien, Muskeln und Nerven; die den vegetativen Verrichtungen dienenden Bindesubstanzen dagegen sollen, entsprechend ihrer verschiedenen physiologischen Dignität, einen morphologisch gesonderten Ursprung haben; sie sollen „parablastischer" Natur sein, und aus dem weissen Nahrungsdotter entstehen. Der functionelle Gegensatz der Bindesubstanzen gegenüber den andern einfachen Geweben wäre durch den verschiedenen Ursprung bedingt; es wäre ferner in diesem die Erklärung für die Thatsache gegeben, dass die Bindesubstanzen niemals Epithelien oder Muskeln, die Epithelien nie Bindegewebe, Knorpel oder Knochen wïrden.

Fand nun auch zunächst diese dualistische, auf physiologischen Gesichtspuncten beruhende Auffassung des Organismus wenig Anklang, so hat sie doch mit dahin geführt, die Annahme, dass Alles, was den Organismus bilden hilft, ein und derselben Quelle entstamme, stark zu erschittern. Es ist mehr und mehr die Aufmerksamkeit auf den Nahrungsdotter hingelenkt worden; die Möglichkeit, dass auch in diesem Theil des Eies Zellen vorhanden seien, oder sich neu bilden könnten, hat man nicht a priori abgewiesen, und es haben in neuester Zeit z. B. allein für das Hühnerei Goet te und Balfour einen Theil der zum Keime hinzutretenden Zellen aus dem Nahrungsdotter wenigstens einwandern lassen. Wenn auch beide Beobachter daraus nicht gefolgert haben, was $\mathrm{H}$ is aus ähnlichen Beobachtungen schloss: Remak's stillschweigende Voraussetzung, dass allein der Bildungsdotter die Bausteine des Organismus liefere, glaubten sie nicht mehr aufrecht erhalten zu können.

Gegenüber diesen Abweichungen von der eine Zeit lang unbestritten herrschenden Lehre Remak's hat nur der letzte Beobachter, Kölliker, mit Entschiedenheit die alleinige Richtigkeit des Remak'schen Standpunctes betont. In ganz besonderem Maasse gilt dies für die Bildung des Blutes, die, mit alleiniger Ausnahme Kölliker's, von jedem Beobachter nach $\mathrm{Remak}$ in einer anderen Weise geschildert worden ist, so dass es beinahe unmöglich scheint, die verschiedenen Angaben auch nur soweit in Uebereinstimmung zu bringen, dass man uber die Hauptpuncte ins Klare kommt. 
Hauptsächlich betreffen die Differenzen die erste Entstehung, die Quelle des Blutes. Deswegen bedürfen die Vorgänge im Keim bis zum Auftreten der Blutmassen und der ersten Blutbahnen einer erneuten Untersuchung. Selbstverständlich mass die Beobachtung von einer genauen Untersuchung der Localität ausgeben, in der das erste Blut sich zeigt, nämlich des $\mathrm{Keim-}$ randes. Wie ist zu Anfang der Bebrütung der Keimrand beschaffen? Wie bildet er.sich um während der Bebrütung, und woher stammen die Zellen, die zu Blut werden? Das sind die zunächst zu beantwortenden Fragen. Da aber auch noch streitig ist, wie sich die Gefässwände zu ihrem Inhalt verhalten, ob beides unabhängig von einander entsteht, oder nicht, so muss auch diese Frage beantwortet werden.

Es wird eine Uebersicht der bisherigen Beobachtungen zwar erweisen, dass die Antworten einander direct widersprechen; sie wird aber auch zeigen, warum die Widerspriiche auftreten mussten. Es hat ein Theil der Beobachter hauptsächlich Durchschnitte, ein anderer hauptsächlich Flächenbilder untersucht; eine geniigende Combination beider Untersuchungsmethoden habe ich bei keinem Beobachter angetroffen.

Remak (1) hat, soweit seine Methoden erlaubten, mit beschränkten Mitteln Erstaunliches geleistet. Er geht vom Studium der Randpartie des Keimes aus; aber die ersten Stadien blieben ihm unbekannt. „Wie der Rand der Keimscheibe sich zum Dotter verhält", sagt er, „konnte ich an unbebrïteten Eiern nicht ermitteln" (pag. 2). Er giebt aber eine präcise gefasste Schilderung des Keimrandes zu Ende des ersten Tages, die ich zum grössten Theil mit seinen eigenen Worten wiedergeben will, da seine Darstellung ein Muster von Bestimmtheit und Klarheit ist.

„Der äussere Umfang des Embryo wird durch den äusseren Rand der Seitenplatten bestimmt. Den jenseits dieses Randes gelegenen Theil der Keimscheibe, welcher aus drei Blättern, dem peripheren Theil des Hornblatts, dem peripherischen Theil des mittleren Blattes und dem peripherischen Theil des Darmdrüsenblattes besteht, nenne ich das Keimlager (pag. 11).

Am Schlusse des ersten Tages befindet sich nur ein kleiner Theil des Keimlagers im Bereich der area pellucida, der grössere im Bereich des Dotters, in der area opaca. Das oberste Blatt breitet sich am schnellsten und weitesten auf der Oberfläche des 
Dotters, dicht unter der Dotterhaut aus; das mittlere Keimblatt folgt zwar dem Hornblatte, bleibt aber an Umfang weit hinter ibm zuräck; das Drüsenblatt hängt am Rande des Fruchthofes so innig mit dem Dotter zusammen, dass es ohne Gewalt nicht von demselben getrennt werden kann."... „Eine membranöse Schicht, die Dotter rinde, ist es, mit weleher das Drisenblatt in Verbindung bleibt. Sie bedeckt den peripherischen Theil der Keimhöhle und geht. nach Aussen hin unmittelbar in den übrigen, freien Dotter über (pag. 14, 15, §§. 23, 25, 26).

Die Dotterrinde besteht ihrer Hauptsache nach aus runden oder ovalen Körpern, welche den Kugeln des übrigen freien Dottergelbs äbnlich sind. Sie unterscheiden sich ron denselben durch geringeren Umfang, grössere Festigkeit und Durehsichtigkeit sowie den Mangel eines feinkörnigen Inhalts. Zwischen ihnen zeigen sich helle, schmale Zwischenräume. In der Nähe des Fruchthofs sieht man statt der isolirten Dotterkugeln Gruppen von kleineren, welche ihnen an Umfang gleichkommen, und in vielen Fällen kann man sich überzengen, dass die hellen Conturen, welche jene Gruppen amgeben, der Lage nach durchaus den Conturen der mit Fetttröpfchen erfüllten Drüsenblattzellen entsprechen". (\$. 27, pag. 16.)

Remak spricht sich also uber den Ort, wo das mittlere Keimblatt aufhört, und über die Art seines Randes nicht aus; auch das Darmdrüsenblatt verliert sich nach ihm in die Dotterrinde, ohne dass er angäbe, wieweit diese als seine Fortsetzung zu betrachten wäre. $\mathrm{Er}$ hält die Kugeln der Dotterrinde indess für Zellen, und glaubt, dass ibr Inhalt durch eine Art von Furchungsprocess zerfiele.

Es wäre aber am Ende des ersten Tages eine gewisse Strecke der area opaca dreiblättrig; das geht anch aus Remak's schematischen Querschnittszeichnungen hervor. (Tafel II, Figur 17. D.)

Entsprechend diesen summarischen, nicht auf histologisches Detail eingehenden Angaben über das Verhalten des Keimlagers ist auch die Schilderung über das Auftreten des Blutes im Lapidarstil abgefasst. Da Remak nach eigener Angabe nicht wusste, „wie die ersten Blutzellen entstehen" (pag. 14), so beschränken sich seine Angaben auf die Bildung der Gefässe. Die Gefässe bilden sich ,im peripherisehen Theile des mittleren Keimblatts ; sie erscheinen als netzförmig verbundene, beinahe undurchsichtige Cylin- 
Die Entstehung des Blutes und der ersten Gefässe im Hühnerei. 549

der"; dieselben werden, "namentlich am Rande des Fruchthofes", zu Canälen, deren Wand eine einfache Zellenlage ist. „Die meisten Gefässcanäle erscheinen inn erhalb des Fruch thofes durchaus leer von Zellen.... Dagegen sind die breiten Gefässcanäle in der area vasculosa in der Regel schon am Schlusse des ersten Tages, bevor noch eine Spur des Herzens sich zeigt, mit zahlreichen, theils farblosen, theils gelbröthlichen granulirten Zellen (Blatzellen) gefüllt." Vermuthlich "verdanken diese den in der Axe der Gefässanlagen befindlichen Zellen ihr Entstehen".

Die Gefässe also legen sich solide an, und werden hohl; die in der Axe liegenden Zellen werden Blut, die peripheren bilden die Gefässwand. Die Gefässe sind also Intercellularr äume. Wie die soliden Cylinder gebildet werden, sagt Remak nicht; das erste Auftreten derselben schildert er aber, ebenso wie ihr späteres Verhalten, viel richtiger als mehrere seiner Nachfolger.

Afanasieff (4) studirte, wie Re mak, hauptsächlich Flächenbilder; er untersuchte aber nicht die area opaca, wo doch das Blut zuerst auftritt, sondern die area pellucida, indem er voraussetzte, dass der Entwickelungsmodus der Gefässe und ihres Inhalts in beiden Gegenden der gleiche sei. Er kommt zu dem Resultate, dass die Blutbildung nicht nur aufdie area opaca beschränkt ist, sondern auch in der area pellucida stattfindet, dass aber keineswegs die Gefässanlagen solide sind.

Er findet nämlich im mittleren Keimblatt blasenartige Gebilde, die vermuthlich durch Hohlwerden einer Zelle oder eines Zellenaggregats entstehen; in der Wand der Blasen finden sich Kerne und „buckelförmige Erhabenheiten". Die Blasenwand treibt Fortsätze nach Innen und Fortsätze nach Aussen; letztere treten mit denen benachbarter Blasen in Verbindung. Zwischen den Blasen bleiben Hohlräume und in diese, die ein zusammenhängendes Canalsystem bilden, kommen die Blutkörper hinein; die letzteren sind nämlich Produete der Blasenwand, die durch Abschnärung frei werden.

Es würden sich also die Gefässe zuerst anlegen; der Gefässinhalt aber wäre als Product der Gefässwand zu betrachten.

Diese Beobachtungen sind lückenhaft; die beschriebenen Blasen sieht man zwar, aber was sie eigentlich bedeuten and wio 
der Anschein einer Blasenbildung entsteht, ist Afanasieff durchaus unbekannt geblieben. Die Frage, wie es denn kommt, dass zuerst in der area opaca das Blut auftritt, hat Afan a si eff gar nicht aufgeworfen und die area opaca fast gar nicht studirt; deshalb steht er seinen Bildern rathlos gegenuiber, er kann sie nicht wegläugnen aber noch weniger deuten.

Es hat auch der nächste Beobachter, His (2) den ron Afanasieff gewählten Weg nicht eingeschlagen; er beginnt mit dem genauen Studinm der Keimperipherie, wobei seine Beobachtungen die von Remak vielfach ergänzen, aber auch in manchen Puncten wesentlich von den früheren abweichen.

Nach $H$ is besteht der Keim des befruchteten, unbebriteten Eis aus einem zusammenhängenden oberen Keimblatt, dem die Anlage des noch unrollständigen unteren Keimblattes in Gestalt verstreuter Zellenhaufen, der ,subgerminalen Fortsätze“, anliegt. Der Rand des Keims ruht auf einem dicken Ringe weissen Dotters, dem „Keimwall“, der in eine dickere innere, eine dünnere äussere Zone zerfällt. In den Keimwall hinein erstrecken sich ebenfalls subgerminale Fortsätze, und auf seiner Oberfläche liegt der Rand des oberen Blattes auf. Erstere vermehren sich im Laufe der Bebriitung, so dass das den Keimwall durchsetzende Zellennetz immer dichter wird; dabei bildet sich an der unteren, dem gelben Dotter zugewandten Keimwallflïche eine zusammenhängende Zellenlage aus, die ebenso den Keimwall vom gelben Dotter trennt, wie das obere Keimblatt ihn von der Dotterhaut scheidet.

Der Keimwall wird also zu einer von Zellen durchwachsenen, $z$ wischen zwei Zellenschichten eingeschlossenen Lage weissen Dotters.

Nach Vollendung der unteren Zellenlage, die His „untere Gränzschicht" nennt, trennt sich die innere Zone des Keimwalls ganz vom Dotter $a b$ und bleibt in Verbindung mit dem oberen Keimblatte. Das untere Keimblatt, das sich unterdessen im Centrum des Keims aus "den subgerminalen Fortsätzen gebildet hat, geht in diese innere Zone des Keimwalls über.

Dieselbe ist also identisch mit der "Dotterrinde" Remak's; sie besteht aber nicht aus "Dotterzellen", wie jener angab und die er genau beschrieb, sondernaus weissem Dotter, in dem Zellennetze sich finden.

Auf der abgelösten Keimwallschichte bildet sich eine zusam- 
Die Entstehung des Blutes und der ersten Gefässe im Hüühnerei. $\quad 551$

menhängende Zellenplatte, das "Gefässblatt". Die Bildung desselben erfolgt nicht durch Zellen allein, sondern unter Betheiligung der Dotterelemente des Keimwalls. Diese selbst ändern sich dabei in doppelter Weise. „Ein grosser Theil der Dotterelemente erfährt zunächst einen Zerfall der Kerne (als solche sieht $H$ is die Inhaltskörper an), welchem entweder eine Lösung der letzteren, oder eine vorherige Lösung der umhüllenden Membran folgt. Im ersteren Falle bilden sich die bekannten Vacuolen, im letzteren kleinere Anhärufungen der freien (freigewordenen) Kerne. Letztere lösen sich nach und nach gleichfalls auf, oder sie fallen den archiblastischen Zellen als Nahrung anheim (S. 75).

Diese regressive Metamorphose betrifft die innersten, oberflächlichsten Dotterelemente des Keimwalls.

Neben den zerfallenden ,findet man im inneren (d. b. abgelösten) wie im äusseren Keimwall eine grosse Anzahl von weissen Zellen (d. h. Dotterelementen), welche je nur einen, oder einige wenige etwas grössere Kerne enthalten. Eine weitere Metamorphose leitet sich damit ein, dass die Kerne dieser Zellen mit feinkörnigem Protoplasma sich umbüllen. So lange sie noch in der ursprünglichen Hülle eingeschlossen bleiben, können sie diese entweder nur sehr partiell, oder auch vollständig ausfiullen" (pag. 76).

Die vollständig ausgefüllten Dotterkugeln enthalten meistens mehrere „Kerne“ und ihr „Protoplasma“ zerfällt in einzelne aneinander haftende runde Ballen, deren jeder einen „Kern“ umschliesst.

Dies sind die Aenderungen der area opaca, welche dem Auftreten des Blutes vorhergehen. Das wesentliche ist die Thatsache, dass in den Theil des Keims, in welchem das Blut zuerst auftritt, weisse Dotterelemente aufgenommen werden. Remak liess das Blut entstehen im peripherischen Theil des mittleren Keimblatts, der seiner „Dotterrinde" aufliegt; $H$ is lässt es in der Dotterrinde, seinem Keimwall, sich bilden und auf die obere Fläche derselben treten. Wenn es dorthin gelangt ist, zeigen sich die schon von Remak beschriebenen soliden Cylinder; aber dieselben liegen nicht im mittleren Keimblatt, sondern in einer Zellenplatte, die unabhängig von demselben sich bildet, dem „Gefässblatt", und sie treten erst in späteren Stadien mit dem mittleren Keimblatt in Verbindung. 
Die Vorgänge, welche die Bildung des Gefässblattes einleiten, welche zum Auftreten der soliden Cylinder Remak's führen, beschreibt $\mathrm{His}$ folgendermassen (pag. 96, 97):

„Es können (in Folge der progressiven Metamorphose derjenigen Dotterelemente, welche wenige Kerne haben) in einer weissen Mutterzelle ganze Conglomerate von Tochterzellen enthalten sein, deren jede aus einem kernhaltigen, einer eigenen Membran entbehrenden Protoplasmaklumpen besteht. Solche Conglomerate besitzen, ihrer Zusammensetzung gemäss, eine höckerige Oberfläche, sie können selbst etwas verästelte Figuren bilden....... Die Protoplasmamasse, welche im Innern der weissen Dotterkugeln des Keimwalls sich entwickelt, zeigt sehr frühzeitig eine gelbliche Färbung.... Wo nun eine weisse Kugel einen bedeutenden Haufen von körnigen Zellen in sich entwickelt, oder wo mehrere solcher Haufen zusammenstossen, da ergiebt sich die Bildung eines röthlichen Fleckens oder einer Blutinsel."

Durch Zusammentreten der so entstandenen Zellhaufen, die anfangs von der Hiulle der Dotterkugel, in der sie entstanden sind, umgeben werden, bildet sich das Gefässblatt. Es geschieht das zunächst ,in den oberflächlichen Schichten des Keimwalls", später kommen die Blutinseln und die Zellen zwischen ihnen auf die obere Fläche des Keimwalls zu liegen.. „Wie die Gefässröhren sich bilden, ist schwer zu sagen; soviel ist sicher, dass zuerst compacte Zellenstränge da sind, in deren Innerem dann durch Auseinanderweichen der Zellenstränge ein enges Lumen auftritt" (pag. 98).

Das Gefässblatt tritt in die area pellucida derart hinein, dass es zwischen das Darmdrisenblatt und die untere Muskelplatte (Darmfaserplatte Remak's) sich einschiebt.

Die Darstellung von $\mathrm{His}$ bringt also eine genauere Bestimmung dessen, was in der „Dotterrinde" enthalten ist. Dieselbe ist ein Gemisch von weissem Dotter und Zellen; der weisse Dotter ändert sich während der Bebrïtung in bestimmter Weise um. $O b$ aber die als progressive Aenderung gedeutete Metamorphose eines Theiles der Dotterelemente richtig erkannt ist, ob dieselben wirklich zu Blutinseln werden, das ist eine Frage, welche die Darstellung von His nicht in befriedigender Weise löst. His hat gar nicht näher untersucht, ob seine Deatung die einzige sei, die sich den beobachteten Vorgängen geben lasse. Es ist dies eine Lï̌cke in den sonst so genauen und sorgfältigen Untersuchungen 
dieses Forschers; es musste unbedingt die erwähnte Frage aufgeworfen werden, da bei vielen Dotterelementen eine regressive Umwandlung mit Sicherheit constatirt wurde, und es a priori nicht wahrscheinlich war, dass gleichwerthige Dinge am selben Orte und unter ganz gleichen Bedingungen, in so verschiedener Weise sich umbilden würden. Auch vermisst man nähere Angaben darüber, was aus den subgerminalen Fortsätzen wird, die den Keimwall durchwachsen.

Neuerdings hat $\mathrm{His}(3)$ alle seine bezüglichen Angaben über die Aufnahme weisser Dotterelemente in den Randtheil des Keims und über deren Umwandlung zu Blutinseln aufrecht erbalten. Die aufgenommenen Dotterelemente, die „Keimwallkugeln" sollen sich in einer Weise umwandeln, ,wie das freie Dotterkugeln nicht thun". „Es treten in ihrem Inneren abgegrenzte Haufen einer feinkörnigen, schwach gelbroth gefärbten Substanz auf, in welcher weiterhin helle Flecke und scharf abgegrenzte Kerne wahrnehmbar werden. Wir haben mit andern Worten Zellenbildung innerhalb der Dotterkugeln." Letzteres folgt noch nicht; denn wenn der Nachweis geliefert wird, dass derartige Dotterformen auch im freien Dotter sich finden, an Stellen, wo es zu keiner Zeit zu einer Zellbildung kommt, so ist das ein directer Beweis dafür, dass die von His geschilderten Umwandlungsstadien etwas anderes bedeuten, als Zellbildung in Dotterkugeln. Auf diesen Punct werde ich ausführlich zurlick zu kommen haben.

Die Frage, ob das Blut aus dem weissen Dotter entstehe, ist von dem nächsten Beobachter, Klein (5) nicht aufgeworfen worden. Derselbe knüpft wieder an die Schilderungen Af'anasieff's an, die er zunächst dadurch vervollständigt, dass er die Genese der von ersterem geschilderten Blasen eingehender studirt.

Nach Klein entstehen dieselben durch Vacuolenbildung in einzelnen Zellen des mittleren Keimblatts; die Vacuolen vergrössern sich und ihre Wand, das Protoplasma der ursprïnglichen Zelle, wird dünner. Dabei theilt sich der Zellkern und seine Stücke rüeken mit zunehmender Vergrösserung der Vacuole weiter auseinander. Auf diese Weise entsteht eine Blase, deren Wand in grösseren Abständen von einander Kerne enthält - Endothelblase. - Diese wird nun dadurch, dass von ihrer Wand Auswüchse nach Innen sich vortreiben, sich abschnüren and in die Blase hineinfallen, za einer zellenhaltigen Kugel - Brutzelle. 
Die hineingefallenen Körper sind Zellen und werden zu Blutkörperchen. Dieselben entstehen also nach Klein auf dem Wege einer endogenen Zellbildnng, in eigenthümlich modificirten Zellen des mittle ren Keimblatts. Wie ordnen sich nun die blutkörperhaltigen, geschlossenen Kugeln zu Gefässbahnen?

Es geschieht das durch Vergrösserung der Brutzellen, Aneinanderlegen derselben, und schliessliche Resorption der einander bertibrenden Flächen, so dass aus Reihen von Hohlkugeln Canäle werden, in denen der Inhalt der Hohlkugeln circulirt. Die Gefässe wären also aus hohlgewordenen Zellen hervorgegangen; -sie wären nicht wie die bisherigen Beobachter angenommen hatten, Intercellularräume, sondern Intracellularräume. Das Blut käme auch nicht, wie Afanasieff angenommen hatte, in die Räume zwischen benachbarten Blasen hinein, sondern läge im Innern der Blasen selbst. Das ist eine wesentliche Differenz gegenüber den Angaben Afanasieff's.

Der Auffassung der Gefässe als intracellulärer Räume hat sich auch Balfour (6) angeschlossen. In den Einzelheiten weicht aber seine Darstellung erheblich von der Klein's ab. Die Vacuolenbildung hat er nicht bestätigen können; der Vorgang bei der Bildung des Blutes und der Blutbahnen ist vielmehr folgender: „Einige Zellen der Darmfaserplatte (splanchnopleure) senden Fortsätze aus; diese vereinigen sich mit denen anderer Zellen, und bilden so ein Netzwerk. Die Kerne der Zellen theilen sich, und vermehren sich sehr sehnell an den Punkten, von denen die Zellfortsätze ausgehen (at the points from which the processes start, their division is especially rapid). Vornehmlich die an diesen Punkten gelegenen Kerne färben sich roth und werden zu Blutkörperchen; die übrigen werden mit sammt dem umschliessenden Protoplasma zu einem Endothelium, das sowohl die Blutmassen als die dieselben verbindenden Protoplasmazitge einhullt (become converted into an endothelium both for the processes and the masses of corpuscles). Die Protoplasmastränge werden flüssig, und dadurch wird das ursprüngliche Zellennetz zu einem Netz von Hohlräumen, gefüllt mit Flüssigkeit und Blutkörperchen."

Die letzteren hätten demnach nur die Bedeutung von Kernen, nicht $\nabla$ on Zellen.

Man sieht, dass weder die Angaben von $H$ is sich mit denen 
Die Entstehung des Blutes und der ersten Gefässe im Hühnerei. 555

Remak's vereinigen lassen, noch auch, dass einer derjenigen Beobachter, die wie Afanasieff, Klein und Balfour von Flächenbildern ausgegangen sind, derart mit seinen Vorgängern ubereinstimmt, dass die Differenzen in der Deutung dagegen zurückträten. Es haben die drei Letzteren sehr ähnliche, wenn nicht die gleichen Bilder vor sich gehabt; die an dieselben geknüpften Folgerungen aber sind so grundverschieden, dass das Verständniss der Entstehung des Blutes und der Gefässe viel mehr erschwert, als gefördert erscheint.

Eine sowohl von Remak und His, als auch von Afanasieff, Klein und Balfour gänzlich abweichende Lehre findet sich in einer gleichzeitig mit Balfour's Darstellung verfassten Abhandlung von A. Goette (7). Dieser Forscher hat den Rand des Keims zuerst ganz richtig geschildert, er hat nachgewiesen, dass die innere Zone des Keimwalls von His, der Dotterrinde Remak's, weder Dotter allein, noch auch ein Gemisch von Dotter und Zellen sei, sondern eine Verdickung der unteren Keimschichte, die Jediglich aus Zellen bestehe, und hat dieselbe, mit dem Namen „Randwulst" bezeichnet. Die Bedeutung dieses Randwulstes für die Bildung des mittleren Keimblattes hat Goette zuerst richtig gewürdigt; ebenso gebührt ihm das Verdienst, die richtige Methode für die Untersuchung der Vorgänge bei der Entstehung des Blutes wieder aufgenommen zu haben, nämlich das Studium von Durch. schnitten des Keimrandes.

Wir müssen etwas weiter ansholen, um über die Veränderungen klar zu werden, die nach Goette's Darstellung im Randtheil des Keims bis zum Auftreten des Blutes vor sich gehen.

Wenn gegen Ablauf des Furchungsprocesses der grösste Theil der Furchungszellen den Keim constituirt, und dieser seine definitive Form, die einer zweischichtigen, in der Mitte dünnen, am Rande verdickten Platte annimmt, so greift die Furchung auf den Dotter unter dem Keim tiber, und producirt dort grosse granulirte Zellen, die Dotterzellen, welche zunächst da liegen bleiben, wo sie entstanden sind, und nicht zum Keim hinzutreten. An der Bildung: des mittleren Keimblattes, die durch Zellverschiebung in der unteren Keimschicht aus dem Randwulst nach der Mitte des Keims hin erfolgt, nehmen die Dotterzellen keinen Antheil; sie treten erst in Function, wenn die Mesoblastbildung vollendet ist.

Ist dies geschehen, so sind die Zellen des Randwulstes dazu 
verbraucht; der Randwulst ist geschwunden, und an seiner Stelle liegt die obere Keimschicht dem unter dem Randwulst gelegenen weissen Dotter, dem „Keimwall", auf. An diesen letzteren stösst der Hypoblast mit verdicktem Rande an; es ist also der Keimwall Goette's identisch mit der "Dotterrinde“ Remak's.

Nun erweitert sich die Keimhöhle; dabei bleibt überall, mit Ausnahme eines Abschnittes der vorderen Peripherie, der Hypoblast mit dem "Keimwall" in Verbindung, und die Keimhöhle schiebt sich unter den Keimwall ein, so dass die obere Keimschicht nach wie vor demselben aufruht.

Der also vom Dotter abgelöste Keimwall hellt sich auf; der Inhalt seiner grossen Dotterkörner erscheint feingranulirt, und es treten Spalten in ihm auf, die ihn in polygonale Felder vom Umfange der grössten Embryonalzellen zerlegen. Dann sieht der Keimwall aus wie eine directe Fortsetzung des Darmblatts; in Wirklichkeit ist dem nicht so, denn er ist nicht etwa in Zellterritorien zerfallen, sondern bleibt nach wie vor Dotter, und die Spaltbildung ist ein Symptom der beginnenden Auflösung seiner Elemente.

In diesem Stadium wandern in den Keimwall die Dotterzellen von der Keimhöhle her ein; sie zerklüften sich, gelangen zwischen die Zellen des sich ausbreitenden Mesoblasten, und wèrden, von diesen umgeben, zu Blutinseln.

Nach Goette wären es also nicht Dotterelemente des Keimwalls, sondern in denselben eingewanderte Furchangszellen, die zu Blutinseln würden; und das ist ein wesentlicher Unterschied gegenüber der Auffassung von His. Mit His stimmt Goette aber darin überein, dass die ersten Blutmassen, die "Blutinseln" im Keimwall entstehen, unabhängig vom Mesoblasten, und dass ihre Verbindung mit dem Mesoblasten eine nachträgliche ist. Ferner stimmen $H$ is und Goette darin uiberein, dass die Anlage des Blutes der Anlage der Gefässe vorhergeht, ganz unabhängig von ihr erfolgt; das ist eine fundamentale Differenz gegenüber den Angaben von Afanasieff, Klein und Balfour.

In Folge der Erweiterung, die unsere Kenntnisse über den Rand des Keims durch die Untersuchungen von $\mathrm{H}$ is und Goette gewonnen haben, ist der Rand zu einem complicirten Gebilde geworden. An die Stelle der Remak'schen Dotterrinde trat, wie wir sahen, der Keimwall von $H$ is; beide Bezeichnungen meinen 
Die Entstehung des Blutes und der ersten Gefässe im Hühnerei. $\mathbf{5 5 7}$

dasselbe, denn sowohl für den Keimwall, als. für die Dotterrinde des bebrïteten Keims gilt als fundamentaler Charakter die Thatsache, dass der peripherische Theil des oberen Keimblatts ihm aufliegt, während das untere Keimblatt (Darmdrüsenblatt) in denselben sich hinein verliert.

Nach Goette muss nun der Keimwall noch weiter zerlegt werden; in den verdickten Randtheil der unteren Keimschicht, den Randwulst, und den Keimwall, d. h. den Ring weissen Dotters, auf dem der Randwulst aufruht, und der über seine Peripherie hinans sich erstreckt. Der Randwulst Goette's ist ein Theil des inneren Keimwalls von His; erst wenn der Randwulst zur Bildung des Mesoblasten verbraucht ist, ruht das obere Keimblatt direct auf derjenigen Partie des Keimwalls, in die der Hypoblast sich fortsetzt.

Diesen Keimwall fasst aber Goette nicht ganz so auf, wie $\mathrm{His}$; nach letzterem ist er ein Gemisch von Zellen und weissem Dotter, nach Goet te besteht er lediglich aus weissem Dotter; die einzigen Zellen, die sich zu einer Zeit in demselben finden, sind von der Keimhöhle hineingewanderte grosse Furchungszellen, die zu Blutkörpern zerfallen.

Eine andere Auffassung macht sich geltend in der unter Kölliker's Leitung entstandenen Dissertation von Hans Virchow (8). Der Randwulst mitsammt dem Keimwall sind ein untrennbares Ganze, und gehören von Anfang an zum unteren Keimblatt, desšen verdickten Rand sie repräsentiren. Dieses Randgebilde bezeichnet Hans Virchow mit Kölliker als „Keimwulst"; der Keimwulst besteht nur aus Zellen, keineswegs aus Dotter und Zellen; die Zellen selbst sind durch ihre Grösse sowie ihr Aussehen ganz merklich unterschieder von den tubrigen Zellen des Keims, und sie bilden sich im Laufe der Bebrütung zum Epithel des Dottersacks um. Der körnige Zellinhalt verdeckt anfänglich die Zellgrenzen; erst nach und nach werden dieselben deutlicher. „Die Randzellen des Keimwulstes sind cylindrisch, doppelt so hoch als breit. Sie enthalten Kerne, die sich mit Vorliebe in den, dem mittleren Keimblatt zugewandten Fuss placiren, und körnigen Inhalt, der zum Theil die Zellgrenzen verdeckt...; Kerne findet man weit hinaus über den Rand des mittleren Keimblatts. . . Zuweilen trifft man am freien unteren Saume rundliche Zellen, an denen eine Membran nicht deutlich zu unterscheiden 
ist, von körnigem Inhalt erftiltt..... Indessen ist es nicht möglich, zellige Gliederung auf grössere Strecken zu demonstriren" (pag. 19).

Bis zum Ende des zweiten Tages ändert sich unter fortschreitender Aufhellnng des Keimwulstes die Grösse der Zellkerne und die Beschaffenheit des Zellinhalts. Derselbe „besteht aus einer schwach körnigen Masse, und nur selten trifft man in den centralen Partien Haufen dunkler Körner, welche Anfangs so dominirend waren. Dagegen wird man beim Fortschreiten auf die peripherischen Theile finden, dass die dunklen Körnerhaufen in demselben Maasse zunehmen, wie die regelmässige Anordnnng der Zellen und die Palissadenform abnimmt" (pag. 20).

Bis zum fünften Tage vergrössern sich die Zellen, und nehmen durch gegenseitigen Druck die verschiedensten Formen an. Die Zellkerne vergrössern sich noch mehr. Die Zellen sind feinkörnig getrübt, vom Inhalt nicht völlig eingenommen, - ob sie eine Membran haben, giebt $\mathrm{Vir}$ ch $o$ w nicht ausdrücklich an, aber das scheint ans dieser Thatsache doch zu folgen - und sie enthalten anch dunkle stark lichtbrechende Körnchen, die sich im Picrocarmin gelb färben.

Kurz, die ganze Schilderung H. Virchow's erweist nicht, dass er wirklich nur Zellen vor sich hatte und nicht etwa ein Gemisch von Zellen und Dotter. Wären es ausschliesslich Zellen, die den Keimwulst zusammensetzen, so ist mir unerfindlich, weshalb dann nicht eine „zellige Gliederung auf grössere Strecken zu demonstriren" sein sollte. Was H. Virchow als Zellinhalt beschreibt, hat Remak als Dotterrinde beschrieben; ans dem Vergleich der Schilderungen beider Beobachter geht klar hervor, dass beide dasselbe gesehen haben. Auf die Art der Blutbildung geht H. Virchow nicht ein; die neuesten Angaben darüber finden sich bei Kölliker (9). Ebenso ist dort die Geschichte des Keimrandes ausführlich besprochen, weshalb ich die Hauptpunkte nochmals darauss hervorheben will.

Der schon von Oellacher gesehene, von Goette genau erkannte verdickte Rand der unteren Keimschicht, der Randwulst Goette's, wird nicht zur Mesoblastbildung verwandt, sondern er bildet sich in Folge der Bebrütung „durch allmälige Umwandlung seiner Elemente und allseitiges Wachsthum in die Breite und Dicke in den Keimwall von His um" (S. 105). Der Uebergang dieses 
Die Entstehung des Blutes und der ersten Gefässe im Hühnerei. $\quad \mathbf{5 5 9}$

„Keimwulstes", wie ibn Kölliker nennt, in den Keimwall geschieht folgendermaassen:

Nach Vollendung der Keimblattbildung reicht oberes und unteres Keimblatt bis zum Rande der Keimhaut, das mittlere Blatt nur bis an die area opaca. Das obere Blatt ist überall einschichtig; seine Zellen sind im Bereiche des Embryo schmal und hoch, und werden gegen den Rand hin, in der area opaca, breiter und platter. Hier liegen sie direct auf dem Keimwulst (daraus geht hervor, dass der Keimwuist identisch ist mit dem Keimwall von $\mathrm{His}$ ) und dieser, durchweg mehrschichtig, ,besteht aus deutlichen, rundlich eckigen Zellen mit schönem Nucleus und grossen Inhaltskörnern (pag. 133). Das im Bereich der area pellucida einfache untere Keimblatt (Entoderma) verdickt sich gegen den Keimwulst zu und geht direct in denselben uber."

Auf dem inneren Rande des Keimwulstes liegt der verdickte Rand dos mittleren Keimblatts. Dasselbe reicht also nur in den Anfang der area opaca hinein; die area pellucida wäre dreiblättrig, die area opaca nur zweiblättrig; dabei überträfe das untere Blatt an Mächțigkeit bedeutend das obere.

Setzt man die Bebrütung fort, so wird der Rand des Keimwulstes dünner (pag. 173-176), je mehr die Keimhaut sich ausbreitet, desto mehr schreitet die Verdünnung gegen die centrale Partie des Keimwulstes fort. Deshalb besteht am Ende des zweiten Tages der Keimwulst nur aus einer einfachen Lage hober Cylinderzellen, in die das Entoderma der area pellucida übergeht. Dabei gehen die Zellen des Keimwulstes folgende Veränderungen ein:

„Die Zellen des Entoderma sind im Bereich des Embryo körnerarm und blass; im Keimwulst dagegen entwickeln dieselben rasch mit dem Vorschreiten der Bebrütung dunkle runde Körper in sich, welche bald die Zellen fast ganz erfüllen in der Art, dass jede Zelle einen grossen dunklen Inhaltskörper und neben demselben noch eine gewisse Anzahl kleinerer entbält. Am zweiten und dritten Tage werden diese Inhaltskörper gelblich und sieht das Entoderma dann wie anhängender gelber Dotter aus (pag. 175, 176).

Der Keimwulst wird einschichtig in Folge der Verschiebung seiner Zellen nach der Peripherie hin.

Mit der Blutbildung hätte also der Keimwulst Kölliker's identisch mit dem Keimwall von His und von Goette, aber inso- 
fern von jenen wnichtig aufgefasst, als er nicht ganz oder vorwiegend aus Dotter, sondern vielmehr nur aus Zellen besteht, gar nichts zu thun. Diese findet a $\mathrm{u}$ ihm statt.

Es breitet sich nämlich im Laufe des zweiten Bebrütungstages das mittlere Keimblatt weiter aus und kommt in die area opaca, wie schon Remak angegeben hatte. In diesem Theil des Mesoderma entstehen die Gefässe als solide Zellenstränge.

„Als zweites Stadium treten nun Hohlgebilde auf, die an ihrer Wand reichliche Zellenmassen enthalten, welche letzteren nach und nach eine immer entschiedener gelbe und dann rothe Farbe annehmen, und nichts anderes als die erwähnten Blutinseln oder B lutpunkte sind." Dieselben lockern sich und treten in die Hohlgebilde, also die Gefässröhren, hinein. „Es ist je doch nicht nur die erste Blutbildang, sondern auch die erste Gefüsbildung auf $d i e$ areavasculosa und einen kleinen Theil der area pellucida beschränkt" (pag. 167).

Wie die Hohlgebilde entstehen, lässt Köllik er unentschieden; ich finde keine genaueren Angaben darüber. Jedenfalls aber betrachtet er dieselben als Intercellularräume, und spricht ganz entschieden aus, dass im mittleren Keimblatt, und durchaus nicht unabhängig von demselben, die Blut- und Gefässbildung stattfinde.

Es bestehen also, wie aus der gegebenen Uebersicht der Literatur erhellt, ganz wesentlich abweichende Ansichten sowohl über die Zusammensetzung des Keimrandes, als auch über die Entstehung des Blutes. Ueber ersteren Punkt haben sich nur diejenigen Forscher ausgesprochen, die vom Studium der Durchschnitte ausgegangen sind; wollen wir ihre Resultate kurz zusammenfassen, so besteht noch Zweifel über folgende Fragen:

I. Woraus besteht der Keimwall?

II. Zu welchem Keimblatt gehört derselbe?

Die erste Frage beantwortet sich wie folgt:

„Der Keimwall besteht ans weissem Dotter" (Goette). „Der Keimwall besteht aus weissem Dotter, der mit Zellen durchsetzt

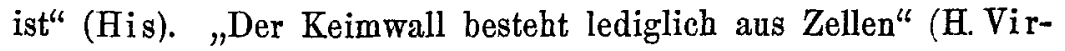
chow, Kölliker).

Auf die zweite Frage haben nur H. Virchow und Kölliker eine präcise Antwort gegeben. 
Die Entstehung des Blutes und der ersten Gefässe im Hühnerei. 561

Nach ihnen gehört der Keimwall, den sie Keimwulst nennen, dem Darmdriisenblatt an.

Goette rechnet den Keimwall iiberhaupt nicht zum Keim, und His theilt denselben keinem Blatte zu.

In Bezug auf die Entstehung des Blutes stimmen aber Kölliker, His, Goette und Remak in einem Hauptpunkt überein; sie geben an, dass das Blut zuerst, und nachträglich die Gefässe erscheinen, dass also keinesfalls die Gefässe zuerst a uftreten und danach der Gefässinhalt. Ferner betrachten sie sämmtlich die Blutgefüsse als Intercellularräume. Ob aber das Blut im Mesoblasten entsteht, wie Kölliker und Remak angeben, oder unabhängig von demselben, wie $H$ is und Goette es schildern, bleibt unentschieden; ebenso ist, wenn letztere beiden Autoren Recht haben, noch streitig, aus welchen Elementen die Blutkörperchen hervorgehen.

Im Gegensatz zu diesen Beobachtern stehen Afanasieff, Klein und Balfour; nach ihnen legt sich erst die Gefisswand an, und das Blut tritt später auf. Das ist aber auch das Einzige, in dem sie übereinstimmen. Jeder schildert die Entstehung der Blutkörper auf eine andere Weise, und nicht einmal darin sind sie einig, ob die Blutgefisse Intercellularriume sind oder Intracellularrïume.

Es wird nun meine Aufgabe sein, die Veränderungen des Keimrandes bis zum Auftreten der Blutinseln und der ersten Gefässanlagen zu verfolgen; dabei werden sich die skizzirten Differenzen über Zusammensetzung und Zugehörigkeit desselben zu dem einen oder andern Keimblatt lösen. Können bis zu dem Punkte, wo die ersten Gefässe auftreten, Durchschnitte zu Grunde gelegt werden: über die Art, wie die Gefässe sich ausbreiten, kann nur das Flächenbild Aufklärung verschaffen. Es werden also die an Durchschnitten gewonnenen Resultate mit den aus Betrachtung der Flächenbilder sich ergebenden zu combiniren sein; und dabei können auch die Angaben derjenigen Beobachter, die ansschliesslich Flächenbilder zum Studium der Blutbildung verwandt haben, auf ihre Richtigkeit geprüft, eventuell anders gedeutet werden.

Der positiven Angabe Kölliker's, dass der "Randwulst" Archr f. mikrosk. Anatomie. Bd. 16. 
Goette's dem unteren Keimblatt zuzurechnen sei, dass er bis zum Ende der Keimblattbildung ind ifferent bleibe und nachher erst durch peripherisches Wachsthum sich zum Epithel des Dottersacks umbilde, habe ich in einem kürzlich publicirten Aufsatze (10) die Behauptung gegenüber gestellt, dass ein Theil der Zellen des Randwulstes zur Bildung des Mesoblasten verbraucht würde, dass also schon während der Keimblattbildung die Verdickung des Randes der unteren Keimschicht eine beträchtliche Veränderung erführe. Den veränderten, wie den ursprünglichen Randwulst rechnete ich zur unteren Keimschichte und habe einige Angaben dariber gemacht, wie sich derselbe nach Vollendung der Keimblattbildung ändert. Es hat nun Kölliker in jüngster Zeit (9, pag. 1009) meine Angaben kurz abgewiesen, indem er ausspricht, ich sei offenbar "durch unvollkommene Präparate getäluscht worden". Das ist eine Vermuthung, kein Argument gegen meine Ausfiblrungen. Auch unvollkommene Präparate können beweisen. Aber wie, wenn meine Präparate nicht unvollkommen sind? Wenn ich seitdem bessere bekommen habe, die evidenter als z. B. meine frìhere Fig. 7 zeigen, dass der Primitivstreif eine Verdickung der unteren Keimschichte ist? Dass nur im Bereiche der Primitivrinne der Epiblast mit dem Primitivstreifen verwächst, hinter derselben aber nicht?

Da meine Angaben über Veründerung des Randwulstes enge mit denen über die Bildıng des Primitirstreifens zusammenhängen, da also, wenn diese unrichtig sind, anch jene fallen miissen, so gebe ich zunächst zum Beweise dafür, dass ich meine früheren Angaben kiber den Primitivstreifen in allen Punkten aufrecht erhalten kann, zwei Abbildungen über den Primitivstreifen. Fig. 1, Quersebnitt des Axenstrangs eines Keims vom Ende des ersten Tages, hinter der Primitivrinne, zeigt den Primitivstreifen scharf vom Epiblasten getrennt; er erscheint als eine spindelförmige Verdickung, in der das untere Keimblatt, der Hypoblast, nur an der Spindelform seiner Zellen als selbständiges Blatt erkannt werden kann. Mit dem Epiblasten hat der Primitivstreif hier gar nichts zu thun; er muss also in der Schicht gebildet sein, die unter dem Epiblasten liegen bleibt, und das ist die primäre untere Keimschicht, die sich in Mesoblast und Hypoblast spaltet.

Fig. 2 zeigt einen Querschnitt durch das hintere Ende der 
Primitivrinne, 9 Schnitte vor Fig. 1; und erst hier beginnt der Epiblast mit dem Primitivstreifen zu verwachsen. Der Primitixstreif kann also gar nicht als eine Verdickung des Epiblasten aufgefasst werden.

Es verhält sieh also der Primitivstreif so, wie ich früher angegeben hatte; und das ist ein Grund mehr für mich, meine die Bildung des Primitivstreifens erläuternden Präparate bei Schilderung. des Keimrandes zum Theil zu Grunde zu legen.

Obgleich die ersten Stadien des Randwulstes und seiner Aenderungen während der Bildung des Mesoblasten schon in meinem erwïhnten Aufsatze (10) beschrieben und abgebildet sind, gebe ich hier, um die Geschichte seiner Entwickelung vollst:indig abhandeln zu können, zwei Abbildungen der ersten Stadien aus der fruheren Abhandlung wieder. Figur 3 (identisch mit Figur 5 der friiheren Arbeit) zeigt den Randwulst des unbebriiteten Keims, einen dicken Zellenring der primären unteren Keimschichte, in den einige feingranulirte Dotterkugeln (Fig. $3 \mathrm{~K}_{\mathrm{II}}$ ) eingelagert sind, Fig. 4 (identisch mit Fig. 7) erklärt, wie sich der Randwnlst bis zur Bildung des Primitivstreifs geändert hat. Er ist in diesem Entwickelungsstadium durch Dotterelemente, die zwischen seinen Zellen liegen, aufgelockert. Am dichtesten stehen die Randwulstzellen zunächst dem Epiblasten, also an der Peripherie der Dotterkugel; je näher dem Centrum derselben, also in den tieferen Randwulstschichten, desto grösser die Zellenabstände, desto reichlicher der interponirte Dotter.

Ein ähnliches Verhalten zeigt schon der Randwulst in Fig. 3, in seine tiefen Schichten sind einzelne Dotterkugeln eingelagert, in den oberen stösst eine Zelle an die andere.

Der Dotter, in den die Randwulstzellen eingelagert sind, geht continuirlich in den zellenlosen Dotter uiber; dieser bildet am erhärteten Präparat den Rand und den Boden der Keimhöhle, und es verliert sich der Hypoblast in ihn hinein (Fig. $4 \mathrm{a}, \mathrm{b}$ ), indem die Spindelzellen aufhören und an Rundzellen anstossen.

In Fig. 3 ist noch eine Gränze zwischen Keimrand und Dotter wahrnehmbar; es trennt sich der Randwulst, die peripherische Verdickung der unteren Keimschicht, einigermassen von seiner aus Dotter bestehenden Unterlage, dem Keimwall. Im Laufe der Bildung des Mesoblasten verwischt sich dieser Unterschied. Der Dotter des Keimwalls gelangt nach oben, zwischen die an ihrem 
ursprünglichen Standorte zurückgebliebenen Zellen des Randwulstes hinein; einer der ersten Effecte der Bebrutung auf die Randpartie des Keims ist also Aufhebung des Unterschieds von Randwulst und Keimwall. Die vorher gesonderten Bestandtheile derselben mischen sich (Fig. 3, R w, K w; Fig. 4, K w). Während also der Rand des unbebrïteten Keims ein Zellenring ist, ist der Rand des bebritteten ein Gemisch von Zellen und Dotter. Für dieses hat His die Bezeichnung "Keimwall" eingefuhrt; dieselbe will ich für den Rand des bebrüteten Keims gebranchen. "Keimwall" und „Randwulst" sind nicht identische Bezeichnungen; ersterer ist eine Modification des letzteren, und daher sind beide Bezeichnungen auseinanderzuhalten.

Der Randwulst gehörte ausschliesslich derunterenKeimschichte an. Verhält sich nun der Keimwall ebenso, oder schliesst er sich an eines der mittlerweile gesonderten Keimblätter?

Um das zu entscheiden, müssen wir noch einen Blick anf die Vorgänge werfen, die die Zellen des Randwulstes so lockerten, dass der unter ihnen liegende Dotter zwischen sie eindringen konnte.

Es hat sich ergeben, dass das bedingende Moment eine Verschiebung der Zellen in centripetaler Richtnng war, wobei in den ersten Stunden der Bebrïtung die Dicke der Mitte des Keims im selben Maasse zunimmt, wie die des Randwulstes abnimmt; dass aber diese Aenderungen der Dimensionen nur die untere Keimschicht betreffen, die obere ganz unberihrt lassen. Es wird also der Randwulst verïndert, lediglich in seiner Zusammensetzung (10, pag. 84-87); die Aenderung ist nur eine quantitative, indem die Dotterelemente vermehrt werden im Verhältniss zur Masse der Zellen. Absolut frei von Dotter ist weder der Rand noch die Mitte des unbebrüteten Keims (Fig. 3, K. I, II). Gehörte also die dotterarme Randpartie zur unteren Keimschichte, so ändert die Vermehrung des Dotters an dieser Zugehörigkeit nichts. Nach wie vor bleibt der geänderte Randwulst bedeckt von der unveränderten oberen Keimschicht, die continuirlich in den Epiblasten der Mitte des Keims übergeht. Was unter der oberen Keimschicht liegen bleibt, gehört der unteren an (Fig. $4, \mathrm{~K} \mathrm{w}$ ).

Nun ist aber, während die obere Keimschicht die Matrix eines Keimblattes darstellt, des Epiblasten, die untere Keimschicht gleichwerthig zwei Keimblättern; aus ihr wird in der 
Die Entstehung des Blutes und der ersten Gefässe im Hühnerei. 565

area pellucida der Mesoblast und der Hypoblast (Fig. 1, Fig. 2). Diese Sonderung der beiden unteren Keimblätter greift auf den Rand des Keims, den Keimwall, noch nicht iiber; darum gehört derselbe nicht einem der unteren Keimblätter allein an, sondern er gehört zu beiden; er hat Zellen die zum Mesoblasten, und andere die zum Hypoblasten zu rechnen sind. Selbstverständlich kann man aber die zum Mesoblasten gehörigen Zellen nicht von den Hypoblastbildnern trennen, beide sind gemischt (Fig. $4, \mathrm{Kw}$ )

Die Thatsache, dass der spindelzellige Hypoblast in den Keimwall hinein sich verliert (Fig. 4, a, b) erweist noch nicht, dass letzterer lediglich eine Fortsetzung des Hypoblasten sei; es reicht in diesem Stadium der Mesoblast noch nicht weit genug nach der Peripherie, um seine Zugehörigkeit zum Keimwall ebenfalls durch directen Uebergang in denselben beweisen zu können. Spätere Stadien zeigen aber evident, dass beide unteren Keimblätter in den Keimwall hineingelangen, und dass nur der Epiblast auf denselben zu liegen kommt (Fig. 8).

Die Zellen des Keimwalls liegen an einer Stelle, an der in frtiheren Stadien eine grosse Anzahl von Randwulstzellen, also Producten der Furchung des Bildungsdotters, sich finden. Es wird daher nicht auf Widerspruch stossen, wenn wir die Keimwallzellen als Abkömmlinge von Furchungszellen betrachten, ebenso wie wir die dariiber gelegenen Zellen des Epiblasten als aus Zellen der oberen Keimschicht hervorgegangen ansehen. Der Umstand, dass die Keimwallzellen zum Theil nicht mehr in directer Continuität mit den übrigen Zellen des Keimes stehen, berechtigt uns durchaus nicht, etwa anzunehmen, dass sie einen eigenen Ursprung hiitten, dass sie vielleicht aus Dotter hervorgegangen wären. Eine derartige Annahme erscheint so unmotivirt, dass sie noch von Niemanden gemacht ist.

Der Gegensatz, in welchem nach dem Vorstehenden der Rand des Keims zur Mitte desselben steht, lässt sich schon an Flächenbildern ganzer Keime erkennen, und schon bei auffallendem Licht. Dem Keimwall entspricht die area opaca, der dreiblättrigen Mitte die area pellucida.

Auch nach Isolirung des Keimes lassen sich bekanntlich beide Höfe unterscheiden, was in Anbetracht ihrer verschiedenen Dicke und Zusammensetzung nicht Wunder nimmt. 
Es ist nun erklärlich, dass bei Isolirung frischer bebrüteter Keime der Keimwall ebenso am Keim hängen bleibt, als der fester gefiugte Randwalst des unbebrïteten. Freilich wird im ersten Fall die Isolirung von Keim und Dotter weniger reinlich ausfallen, als im letzteren, da einzelne Keimwallzellen wohl inımer im umgebenden Dotter stecken bleiben. Die untere Fläche des Keimwalls wird vorwiegend Dotter zeigen; die dort etwa befindlichen Zellen werden von Dotterkörnern verdeckt, und ohne Zusatz von Reagentien, die ihre Kerne hervortreten lassen (etwa Essigsäure) sind sie überhaupt nicht von Dotterkugeln gleicher Grösse zu unterscheiden.

Diese Erwägungen erklären vollständig die Angabe von His, dass der Keimwall ein Ring weissen Dotters sei ; die Untersuchung frischen Materiales muss zu diesem Ausspruch führen. Den grossen Zellenreichthum des Keimwalls erkennt man nur an Durchschnitten von Keimen, die in Chromsäure gehärtet und dann gefürbt sind; Osmiumpräparate zeigen die Zellen im Keimwall nicht, da der dunkel gefärbte Dotter die Zellen völlig verdeckt. Demungeachtet hat $\mathrm{His}$ vollständig erkannt, dass der Keimwall Zellen enthalte; die spätere Angabe von Klein, dass der Keimwall nur Dotter sei, ist unbegriindet.

Ebenso unbegründet ist es aber anch, die Anwesenheit von Dotterelementen im Keimwall iberhaupt in Abrede zu stellen, und selben lediglich aus grossen Zellen bestehen zu lassen, wie Hans Virchow and Kölliker thun. Gleichfalls ist es nicht motivirt, den Keimwall nur zum Hypoblasten zu rechnen.

Gehen wir nun zur Betrachtung der Veränderungen des Keimwalls während des ersten Tages über.

Schon früher (10, pag. 87) habe ich hervorgehoben, dass der Keimwall in eine lockere innere, eine festere äussere Zone zerfällt (Fig. 4, p z n, dz $\mathrm{n}$ ), die ich als proximale und distale Zone benannte. Wenn ich im Folgenden nun auch vorwiegend die Veränderungen der proximalen Zone schildern will, da nur in dieser es zur Blutbildung kommt, so ist die distale Zone ganz dazu geeignet, über einzelne Punkte Aufklärung zu geben, die zu entscheiden das Studium der proximalen Zone allein nicht völlig ausreicht. Besonders ist das der Fall im Laufe des ersten Tages; hier werden wir ab und zu die distale Zone heranziehen mïssen, während wir dieselbe später ausser Acht lassen können, da ihre Veränderungen mit der Blatbildung nichts za thun haben. 
Es ist Figur 5 die genaue Wiedergabe eines Schnittes durch den Keimwall eines 11 Stunden bebrüteten Keims senkrecht auf die Lüngsaxe des Embryo geführt; der Schnitt ist hinter die area pellucida gefallen, er zeigt nur die beiden Keimschichten des Keimwalls, und ihr gegenseitiges Verhältniss bei schwacher Vergrösserung. Fig. 6 und Fig. 7 stellen einzelne Partien dieses Schnittes bei stärkerer Vergrösserung dar; Fig. 6 zeigt die distale Zone, Fig. 7 ein Stiuck der proximalen. (Vgl. Fig. 5 A, B.)

Gegenüber dem früheren Stadium (Fig. 4) zeigt sich wenig Aenderung. Die obere Keimschicht erscheint als eine continuirliche einfache Zellenlage, die untere ist viel dicker, aber die Zellen grenzen nur an den Rändern des Schnitts dicht aneinander, während die Mitte die Zellkerne durch eine Masse körnig erscheinenden Dotters hindurch erkennen lässt (Fig. 5). Ferner ist nur die obere Keimschicht röthlich gefärbt; die viel mächtigere untere erscheint blass, was besonders die Betrachtung mit einem schwächeren Systeme sofort vor Augen bringt.

Bei stärkerer Vergrösserung tritt nun der Bau des Keimwalls viel prägnanter hervor. Die distale $Z_{0}$ ne (Fig. 6) erinnert an den Randwulst des unbebrüteten Keims; in der oberen Keimschicht grenzt eine Zelle an die andere; die Zellen sind rundlich oder auch cubisch, besitzen einen grossen, scharf hervortretenden Kern mit deutlichen, meist mehrfach vorhandenen Kernkörperchen, und da nur die letzteren sich lebhaft färben, ist man anf den ersten Blick versucht, diese für die Kerne, und die Kerne selbst für die Zellen zu halten. Bei genauerem Hinsehen sieht man aber um jeden Kern einen zweiten Contur, die Grenze des Zellprotoplasma (Fig. $6 \mathrm{Ep} \mathrm{b}$ ). Dass auch die Zellen der unteren Keimschicht denselben Typus haben, davon kann man sich in der distalen Zone des Keimwalls am leichtesten iiberzengen (Fig. $6 \mathrm{Z}$ ). Man siebt zuerst nur Kernkörperchen und Kerne; dieselben sind in mehrere Lage uibereinander geordnet, so dass man die viel grössere Mächtigkeit der unteren Keimschicht daraus abnehmen kann; die Grenzen der Zellenleiber, die zweiten Conturen um die Kernkörperchen, sieht man aber viel schwerer. Dieselben sind verdeckt, indem stark glänzende, gelblich aussehende Körner tiber die ganze untere Keimschicht verstreut sind; dieselbe erscheint als eine körnige Masse, in die Kerne in grosser Anzahl eingelagert sind.

Nun stehen aber diese Körner nicht überall gleich dicht; und 
da wo sie locker gesäet sind, was besonders am äussersten Rande und an der unteren Gränze des Keimwalls der Fall ist, treten die Zellgränzen hervor (Fig. $6 \mathrm{Z}$ ). Die Zellen erscheinen hier von derselben Grösse und Beschaffenneit wie in der oberen Keimschicht; nur ist ihr Protoplasma blass geblieben. Dasselbe bricht das Licht viel schwächer als die Dotterkörner; deshalb sieht man die Zellgrenzen nur da, wo kein Dotter auf und zwischen den Zellen liegt. Sieht man aber mehrere Zellen der unteren Keimschicht in der distalen Zone des Keimwalls an verschiedenen Stellen, und findet man, dass selbe ganz gleich sind denen der oberen Keimschicht, so muss man annehmen, dass alle hier sich vorfindenden Zellen ebenso gebaut sind, wenn man nur eine genügende Erklärung dafür zu geben weiss, dass man an den meisten Stellen die Grenzen nicht sieht.

Dagegen scheint nun die Beschaffenheit der proximalen Zone des Keimwalls zu sprechen (Fig. 7). Es ist dort nämlich die untere Keimschicht von Linien durchzogen, welche die granulirte Masse mit den eingestreuten Kernen in kuglige oder polygonale Räume abtheilen. Diese letzteren erscheinen um so eher als die Zellgrenzen, als sie meistens einen Kern enthalten. Wir hätten also hier, in der proximalen Keimwallzone, grosse Zellen, viel grösser als die der oberen Keimschicht; sie besässen einen körnigen Inhalt, der das Licht stark bricht, und glichen also in nichts den Zellen der distalen Zone, auf deren feines, blasses Zellprotoplasma wir schon oben aufmerksam gemacht haben. Diese Zellen wären identisch mit den von H. Virchow und Kölliker beschriebenen grobkörnigen Zellen des Keimwulstes.

Es wäre aber sehr merkwtirdig, wenn der Keimwall zwei so verschiedene Zellenarten enthalten sollte; kleinere blasse in der distalen Zone, viel grössere, mit grobkörnigem glänzenden Inhalt in der proximalen; es wäre sehr auffallend, dass gerade die Zellgrenzen zuerst sich abheben und förmlich wie Membranen erscheinen, während bei allen übrigen Embryonalzellen die Ränder am wenigsten hervortreten. Es ist nun nicht mehr als gerechtfertigt, eingehender zu untersuchen, ob diese erwähnten Linien wirklich die Zellgrenzen sind, oder nicht.

Zunächst ist zweierlei gegen ihre Deutung als Zellgrenzen geltend za machen; einmal die Grösse der umschlossenen Zellterritorien; ferner der Umstand, dass sehr viele der deutlich 
Die Entstehung des Blutes und der ersten Gefässe im Hühnerei. $\quad 569$

umgrenzten Räume mit grobkörnigem Inhalt kernlos sind (Fig. 7 D k I); wie schon erwähnt, wäre es sehr auffallend, wenn binnen zweier Stunden ein Theil der Keimwallzellen, die in Fig. 4 alle gleich gross erscheinen, so eminent an Volumen gewonnen haben sollten, und zwar nur ein Theil. Wenn einmal die Zellen an einem bestimmten Orte wachsen, wie z. B. die Zellen des Epiblasten im Bereich der Rückenfurche, so pflegen doch alle sich zu vergrössern, und nicht nur ein Theil. Viel mehr ins Gewicht fälltaber der Umstand, dass gerade die am deutlichsten sich ausprägenden "grobkörnigen Zellen" keinen Kern haben (Fig. $7 \mathrm{Dk}_{\mathrm{I}}$ ).

Eine jede, gut umgrenzte Zelle, deren Grenze man deutlich sieht, muss den Kern zeigen, besonders wenn man gut gefärbte Schnitte hat.

Die Annahme, dass der Inhalt in diesen Fïllen den Kern verdeckt, widerlegt sich dadurch, dass man durch die dichtesten Körnermassen genug Kerne sieht; hier sind die Körner spärlicher vertreten, und doch ist kein Kern da (Fig. $7 \mathrm{Dk}_{\mathrm{I}}$ ).

Auch der Annahme, dass da, wo eine Zellgrenze ohne Kern erscheint, die Zelle entzwei geschnitten ist, so dass die den Kern enthaltende Hälfte durch das Messer entfernt wurde, wird sich wohl Niemand anschliessen; es müsste also der Kern da sein, wenn ïberhaupt das fragliche Gebilde eine Zelle wäre. Hier fehlt aber mehrfach der Kern; also ist das betreffende kuglige Gebilde keine Zelle. Was ist es aber denn? Die Antwort fällt nicht schwer. Es ist eine durch Chromsäure mehr oder weniger veränderte, grobgranulirte Dotterkugel, deren Rindenschicht deutlich sichtbar ist und als die Grenze einer Zelle mit grobkörnigem Inhalt erscheint. Die Eigenschaften dieser Dotterelemente, ihre Bedeutung und ihre Reactionen habe ich früher schon eingehend besprochen, und verweise darauf (10, pag. 75, 76). Grösse, Gestalt, Form und optisches Verhalten der Inhaltskörner, Alles ist bei den ,grobkörnigen Keimwulstzellen ${ }^{66}$ genau wie bei den "grobgranulirten Dotterkngeln"; beide Bezeichnungen sind identisch. Mit Zellen aber haben die Dotterkugeln nichts zu thun.

Es wird also zellige Gliederung des Keimwalls durch die grobgranulirten Dotterkugeln vorgetäuscht. Wenn das wahr ist: wo sind denn die wirklichen Zellgrenzen? Wenn die Zellen im 
Keimwall nicht gross und grobkörnig sind, wie sehen sie in der That aus?

Sie gleichen in allen Stücken den Zellen der distalen Zone; das blasse Zellprotoplasma kann man anch in einigen Fällen sehen, wenn die Dotterkörner dünn gesäet sind.

Wer den zweiten Contur um die Kernkörperchen, die eigentliche Zellgrenze, sucht, wird ihn an giunstigen Stellen diinner Schnitte immer finden; er wird sich überzeugen, dass in Bau und Grösse kein Unterschied zwischen den Zellen der beiden Keimwallzonen existirt (Fig. 7, Z).

Die ungleichmässige Vertheilnng des Dotters im Keimwall, sowie der Umstand, dass die Dotterkörner bald frei, bald in Kugeln eingeschlossen sind, ist leicht zu erklären. Schon früher habe ich nachgewiesen, dass die Einwirkung der Chromsäure daran Schnld ist. Diese zerstört viele Kugeln, andere lässt sie intact, andere wieder entleeren ihren Inhalt zum Theil, und zeigen dann Hohlriume; dadurch wird ein grösserer oder geringerer Theil des Inhalts der Dotterkugeln frei, bleibt aber im Keimwall liegen.

Den stricten Beweis für die Richtigkeit meiner Angabe, dass die Keimwallzellen kleine Zellen mit grossem Kern nnd einem dünnen Mantel von Protoplasma seien, and nicht grosse, grobkörnige Gebilde, können nur Isolationspräparate liefern; den Beweis für die Richtigkeit der Deutung der grossen, grobkörnigen Elemente als Dotterkugeln muss ihre weitere Entwicklung, sowie ihre Reaction, der Erhärtung gegeniuber, beibringen.

Was den ersten Punct anbetrifft, so habe ich sehr viele Isolationspräparate aus jïngeren Keimwällen gemacht, und alle haben mir die Zellen so gezeigt, wie günstige Stellen meiner Durchschnitte (Fig. 21, a, b, e).

Das Studium älterer Keimwälle, die heller erseheinen und darum leichter aufzulösen sind, an Schnitten beweist, dass die Keimwallzellen so bleihen, wie sie um die Mitte des erstên Bebrïtungstages sind (Fig. 9, 10, 20).

Isolationspräparate von erhärteten Keimwällen hat meines Wissens noch Niemand angefertigt, wenigstens keine Abbildung von isolirten Zellen aus dem Keimwall gegeben; deswegen will ich hier meine Isolationsmethode kurz besprechen. Es kam darauf an, die area pellncida ganz zu entfernen, nur Bestand- 
Die Entstehung des Blutes und der ersten Gefässe im Hühnerei. $\mathbf{5 7 1}$

theile des Keimwalls, und nichts anderes zu erhalten; deshalb sah ich von der Untersuchung frischer Keimwälle ab, da man leicht Bruchstücke der oberen Keimschichte bekommen, und abgerissene Zellen derselben für Keimwallzellen halten kann. Ausserdem sind frische Zellen viel schwerer vom Dotter zu unterscheiden als gehärtete und gefärbte; und da ich die Gestalt der Zellen in gehärteten Keimwall eruiren wollte, wählte ich bereits fertige Schnitte, um die Zellen des Keimwalls zu isoliren.

Es wurde die area pellucida mit der Scheere entfernt, und deren vollständige Entfernung durch das Mikroskop constatirt; dann wurde der überflüssige Dotter entfernt, und der möglichst isolirte Keimwall in Ranvier's diluirtem Alkohol oder in 10\% Kochsalzlösung mehrere Wochen $(3-5)$ macerirt. Aus diesem kamen die gelockerten, aber noch zusammenhängenden Keimwallstücke in absoluten Alkohol, und wurden in Nelkenöl erst in toto angesehen, dann zerzupft. Die obere Keimschicht lkann man sehr leicht in toto abreissen; die tieferen Keimwallschichten zerfasert man mit Nadeln, und bekommt unschwer einzelne Zellen zu Gesichte, die nur aus dem Keimwall, nicht anderswoher stammen können. Dadurch, dass man unmittelbar vor dem Zerzupfen das Präparat noch ansehen kann, ist jeder Irrthum über die Herkunft der isolirten Elemente auszuschliessen.

Die isolirten Zellen zeigten oft Andeutungen ron feinen Ausläufern (Fig. $21 \mathrm{a}, \mathrm{b}, \mathrm{c}$ ); auch an Schnittpräparaten älterer Keimwälle bekam ich dergleichen zu sehen (Fig. 10 Z). Es kann dies aber eine Folge der Schrumpfung sein, die beim Erhärten wohl nicht zu vermeiden ist.

Sind also die grobkörnigen Elemente des Keimwalls und seine Zellen wohl auseinander zu halten: den Beweis dafür, dass erstere Dotter sind, keine andere Bedeutung haben als die Elemente einer beliebigen Stelle des gelben Nahrungsdotters, muss die weitere Entwicklung derselben bringen. Das Characteristische an den grobgranulirten Dotterkugeln ist das, dass sie in einer Metamorphose zu feinkörnigen Elementen begriffen sind, und um so weniger resistent bleiben, je mehr ihr Inhalt dem der gelben Dotterkugeln gleicht. Sie werden also in mannigfacher Weise während der weiteren Bebrïtung ibr Aussehen ändern; aber sie werden weniger ibre Form gegenüber der Chromsäureeinwirkung beibehalten. Nun sieht man auch nicht nur die verschiedensten 
Grade der Füllung an ihnen; man sieht nicht nur zunehmende Gelbfïrbung des Inhalts; man trifft sogar mitunter massenhafte Ansammlungen durchaus leerer Kugeln an (Fig. $13 \mathrm{Dk}_{\mathrm{I}}$, $\mathrm{Dk}_{\mathrm{II}}, \mathrm{Dk}_{\mathrm{II}}$ ).

Sie werden zu Vacuolen; und das geschieht nur Dotterelementen bei Zusatz von solchen Reagentien, die zum Härten geeignet sind, wie ich früher $(10,1$. c.) des Nüheren auseinandergesetzt habe.

Es bleibt nun noch ein Punct zu erledigen. Wie liegen die Zellen des Keimwalls zu dessen Dotterelementen?

Da letztere die Zellen an Volumen ganz bedeutend übertreffen; da sie ferner kuglig sind, so werden zwischen ihnen iiberall Rüume ausgespart bleiben, in denen Platz genug für die Zellen des Keimwalls ist. In diesen Interstitien finden sich die Keimwallzellen; in ihnen verschieben sie sich bei dem Wachsthum des Keimwalls. Das zeigt klar der Flächenschnitt (Fig. 20). Ein Durchschnitt kann das natiurlich nicht so hervortreten lassen; auch die bisher uur angewandte Flächenansicht ganzer Keimwälle ist untauglich dazu. Die Dotterelemente erscheinen hier, Fig. 20, ganz so wie in Fig. 13, obgleich beide Keime einen Unterschied von 20 Stunden in der Entwicklung haben.

Ich glaube in Vorstehendem den Nachweis geliefert zu haben, dass man den Keimwall als ein Gemisch von Zellen und Dotterelementen ansehen muss, und dass erstere in den Interstitien der letzteren liegen. Keinesweges besteht der Keimwall aus Dotter allein wie Goette angab; noch weniger aber kann ich mich an Kölliker und $\mathrm{H}$. Virchow anschliessen, die ihren „Keimwulst", lediglich aus grossen, grobkörnigen Zellen bestehen lassen. Was diese Beobachter für Zellen halten, ist Dotter; die wirklichen Zellgrenzen hat wahrscheinlich der körnige Dotter ihnen verdeckt. Die Ansicht von His über die Zusammensetzung des Keimwalls muss ich nach all meinen Resultaten, aus Durchschnitten, Flächenschnitten und Isolationspräparaten gewonnen, lediglich bestätigen, und berufe mich dafür auf Fig. 4, 6, 7, $9,10,13,20,21$.

Ist nun aber die Ansicht von His ebenfalls begründet, dass. ein Theil der Keimwallzellen von den Dotterelementen abstamme? $\mathrm{His}$ schliesst dies aus Bildern, die ihm Kugeln mit grösseren Kernen und feinkörnigem Protoplasma um die Kerne herum zeigen; 
wenn $\mathrm{Hi}$ is auch derartige Bildungen aus späteren Stadien beschreibt, sie finden sich schon sehr früh, um die Mitte des ersten Tages, und wenn sie wirklich Vorläufer von Zellen sind, so tritt diese Zellbildung aus weissem Dotter früher auf, als $\mathrm{His}$ angegeben hat. Schon Fig. $8 \mathrm{Dk}$ zeigt derartige Dotterkugeln mit grösseren Kernen und feinkörnigem Inhalt um die Kerne; sind das wirklich Dotterelemente in progressiver Metamorphose?

Haben sie diese Bedeutung, so dürfen sie sich nur da finden, wo Zellen liegen, und im freien Dotter nicht vorkommen; sie dürfen dann ferner nicht mit denjenigen Dotterelementen, deren Inhalt ganz sich löst, die in regressiver Umwandlung begriffen sind und ,den archiblastischen Zellen als Nahrung anheim fallen", durch Uebergangsstufen verbunden sein; endlich diirfen sie durch Reagentien nur so verändert werden, wie die Zellen des Keims; die Kerne müssen sich auch gerade so verhalten, als die der Keimzellen.

Es treffen nun aber alle diese Forderungen nicht zu. Sowohl die Uebergangsstufen zu Elementen, die in Zerfall begriffen sind, lassen sich in Masse auffinden, als anch im freien Dotter, sogar in unbefruchteten Eiern, Dotterkugeln mit grössern und kleinern Inhaltskörpern, also mit Andeutnng einer endogenen Zellbildung, jederzeit anzutreffen sind (Fig. $22 \mathrm{c}_{\mathrm{I}}, \mathrm{d}$.)

Die Reaction der grösseren, von His als Kerne gedeuteten Körner gegen Farbstoffe, z. B. Carmin, ist eine ganz andere, wie die der Zellkerne; und alle die in "progressiver Metamorphose" befindlichen Dotterkugeln ändern sich auf Chromsäurezusatz derart, dass sie kaum wiederzuerkennen sind. Ist es nun wahrscheinlich, dass Reagentien, die die fertigen Zellen so vorzüglich conserviren, die werdenden in den meisten Fällen total zerstören? Ist es denkbar, dass ein Kern im Status nascendi einen Farbstoff nicht aufnimmt, den ein fertiger Zellkern stets imbibirt, dass er dagegen in Osmium sich tief schwärzt, während dieses den fertigen Zellkern intact lässt?

Auch in Aussehen unterscheiden sich die "Kerne" der Dotterkugeln bedeutend von den Zellkernen. Diese haben meistens mehrere Kernkörperchen, jene sind homogen; diese sind blass, jene glänzen sehr stark. Deshalb ist es meines Erachtens unmöglich, beiderlei Gebilde, nämlich die grösseren Körner der Dotterkugeln und die Zellkerne, als gleichwerthig anzusehen. 
Das Protoplasma der Keimzellen verhält sich ebenfalls anders gegen Chromsäure und Osmium, sowie gegen Picrinsäure, wie der feinkörnige Inhalt der Dotterkugeln, den $\mathrm{His}$ einfach als Protoplasma bezeichnet. Alle diese Reagentien lassen das Zellprotoplasma unverändert; der Inhalt der Dotterkugeln dagegen wird in Osmium dunkel, bei längerer Einwirkung schwarz, gerinnt unter Einwirkung: von Chromsäure und wird dabei gelblich, und färbt sich intensiv gelb in Picrinsäure, sowie in Picrocarmin. Die Dotterkugeln des Keimwalls verhalten sich in diesen Reactionen wie die grobkörnigen Kugeln im gelben Dotter.

Es liegt also kein Grund vor, anzunehmen, dass eine progressive Metamorphose für einen Theil der „Keimwallkugeln“, wie His neuerdings die grossen Dotterelemente bezeichnet hat, Geltung habe; die Gruppirung des Inhalts kann manchen entfernte Aehnlichkeit mit Zellen geben, aber die Keimzellen sehen ganz anders aus, wie die Inhaltsballen der Dotterkugeln und reagiren in durchaus verschiedener Weise.

Was His abgebildet hat (2, Taf. X, Fig. 4. 3, Taf. XIII, Fig. 9 bis 19) ist ganz und gar ähnlich Dotterstadien, die man aus dem gelben Dotter unbefruchteter, oder auch während der Bebriitung abgestorbener Eier erbïlt (Fig. 22).

Auch im freien Dotter kommen aus Segmenten zusammengesetzte Bildungen vor (Fig. $22 \mathrm{a}, \mathrm{b}, \mathrm{c}, \mathrm{d}$ ); und die einzelnen Segmente können ungleichmässige Stufen einer körnigen Differenzirung zeigen (Fig. 22, a, b); sind sie alle feingranulirt geworden, so liegen oft noch ein oder mehrere grössere Inhaltskörner da, die an Kerne in fein granulirtem Protoplasma erinnern können, aber das Alles bedeutet doch noch keine Zellbildung! (Fig. 22d.) Die gezeichneten Dotterstucke sind aus dem gelben Dotter eines abgestorbenen Eies entnommen, möglichst weit vom Keim entfernt; sie können unmöglich Zellen sein oder werden.

Aus dem Gesagten geht wohl hervor, dass derartige Dotterformen, wie sie $\mathrm{H}$ is beschrieben und als Zellbildungsstadien gedeutet hat, weder ausschliesslich auf den Keimwall beschränkt sind, noch auch unmittelbar vor Bildung der Blutinseln sich finden, sondern schon bedeutend frïher vorkommen. Da sie aber auch in solchen Eiern anzutreffen sind, in denen es niemals zur Zellbildung kommt, weil sie unbefruchtet sind; 'da sie ferner im 
Die Entstehung des Blutes und der ersten Gefässe im Hühnerei. $\quad \mathbf{5 7 5}$

abgestorbenen Ei sich ebenso gut finden als im lebenden; da sie auch an Orten vorkommen, wo sich zu keiner Zeit Zellen finden: so müssen sie eine andere Bedeutung haben, als $H$ is ihnen zuertheilt; Kugeln, die im Begriff sind, zu Zellen zu zerfallen, können sie nicht sein. Die Theorie von His, dass ein Theil der weissen Dotterkugeln Zellen producire, ist unhaltbar; ein Nebenkeim existirt nicht, es werden ebenso wenig im Keimwall Zellen in Dotterelementen erzeugt, als anderswo.

Für die Richtigkeit dieses Satzes wird das Folgende die positiven Grïnde beizubringen haben. Vorher wollen wir noch das Verhältniss des Keimwalls zur area pellucida auf diesem Entwicklungsstadium betrachten.

Fig. 8 ist ein Stiuek eines Schnittes, der um 10 Schnitte vom hinteren Ende des Primitivstreifens entfernt ist. Der Keimwall erscheint als beträchtliche Verdickung, in die alle Zellen der area pellucida, die unter dem Epiblasten liegen, sich hinein erstrecken. Nur der Epiblast setzt sich auf den Keimwall fort (Fig. 8, E p b).

Die Zellen des letzteren stehen anfangs sehr dicht; gegen die Peripherie des Keims hin werden sie auseinander gedrängt, und bei im ganzen gleichbleibender Dicke des Keimwalls ist der Zellreichthum desselben am grössten an der Grenze der area pellucida und opaca. Die Dotterelemente zeigen hier viele Stadien ihrer regressiven Metamorphose. Von ein- and mebrkörnigen Kugeln bis zu feingranulirten (vgl. 10, pag. 70-74, Fig. 1) finden sich Uebergangsformen; auch das Stadium, das A. Goette als grosse Furchungszelle gedeutet hat, auf dem Boden der Keimhöhle entstehen und von da in den Keimwall einwandern lässt, ist mehrfach vertreten ( $F i g .8 \mathrm{D} \mathrm{k}$ ).

Goette hatte aus derartigen Bildern geschlossen, dass die eingewanderten Kugeln zu Blut würden, indem sie, im Keimwall angelangt, in Zellen zerfielen. Schon der Umstand, dass sie gelegentlich einen ganzen Tag vor Auftreten der Blutinseln im Keimwall gefunden werden, spricht gegen diese Ansicht. Es sind Dotterelemente, keine Zellen; die eine hat ein grösseres Inhaltskorn, das aber mit einem Zellkern nicht wohl verwechselt werden kann.

Die Vertheilung der Keimwallzellen im Dotter zeigt die Fig. hinlänglich; auch die Variabilität der Dotterformen geht ohne weitere Beschreibung aus der Zeichnung hervor.

Auch auf den nächstfolgenden Entwicklungsstufen, während 
der Dauer der zweiten Hälfte des ersten Tages, bleibt die Zusammensetzung des Keimwalls, und sein Verhältniss zur area pellucida, annähernd so, wie in Fig. 8. Es ändern sich nur seine Dimensionen, da der Keimwall sowohl der Fläche als der Tiefe nach sich vergrössert. Dabei wird der Unterschied zwischen seinen beiden Zonen allmählich geringer, und der Zellreichthum gleicht sich aus, da besonders in der proximalen Zone die Anzahl der Zellen bedeutend zunimmt. Die Vermehrung erfolgt wohl nur durch Theilung der vorhandenen Zellen; dafür spricht der Befund von zalfireichen Theilnngserscheinungen, sowie die eigenthtimliche Ungleichmässigkeit in der Vertheilung der zelligen Elemente. Dieselben stehen nämlich nicht einzeln, sondern in Gruppen; Zellhaufen wechseln mit Haufen von Dotterkugeln ab. Liegt eine Zellanhäufung in der Nähe des Epiblasten, so erbält man den Eindruck, als sei von diesem aus ein Zellenzapfen in den Dotter eingetrieben; wohl deshalb hat $\mathrm{His}$ angegeben, der Keimwall werde von subgerminalen Fortsätzen durchwachsen.

Am besten treten die Zellengruppen hervor an der unteren Grenze des Keimwalls; dort stehen sie am wenigsten dicht, und fallen leicht als isolirte Massen ins Auge (Fig. $10 \mathrm{Z} \mathrm{g}$ ). Man beobachtet hier wie iberall, zahlreiche Theilungsphänomene (Fig. 9, $\mathrm{Z}_{\mathrm{I}}, \mathrm{Fig}$. 10).

Es wird jeder zugeben, dass die Theilung der vorhandenen Zellen genügt, um das Wachsthum des Keimwalls und die Zunahme seines zelligen Materials zu erklären; die Zellen können sich um so Ieichter vermehren, als sie ganz von Nahrungsmaterial umgeben sind.

Die ungleichmässige Vertheilung der Zellen bewirkt, dass der Keimwall, von der Fläche gesehen, eine netzförmige Zeichnung darbietet (His). Da die obere Keimschicht nur eine Zelle dick ist, so sieht man durch dieselbe entweder den anliegenden Dotter, oder anstossende Zellengruppen hindurch. Die Zellgruppen zeigen sich oft durch Zellreihen verbunden; es kommt zu Bildung von Zellringen, die Dotter einschliessen. An Osmiumpräparaten treten solche Zellringe deutlich herror; denn sie bilden da, wo sie dem Epiblasten anliegen, Verdickungen desselben, und diese brechen das Licht anders, als seine dünnen Stellen, so dass sie zuerst ins Auge fallen.

Sieht man nun mehrere, verschieden grosse Zellringe, in 
Die Entstehung des Blutes und der ersten Gefässe im Hühnerei. $\quad \mathbf{5 7 7}$

denen entweder Dotter, oder auch leere Dotterkugeln liegen, so kann man zu der Ansicht kommen, man habe Vacuolen vor sich mit kernhaltiger Wand; wenn ein Zellenring ausser Dotter Zellen einschliesst, so kann man an eine Abschnürung derselben von der Wand denken. Derartige Bilder müssen Klein veranlasst haben, seine „Endothelblasen" und „Brutzellen“ aus hohlgewordenen Zellen abzuleiten; Klein hat diese mit später auftretenden Zellringen in Beziehung gebracht, die zugleich mit der Bildung der Blutgefässe erscheinen. Wir werden auf diese Bildungen speciell eingehen, und dabei wird sich zur Genuge herausstellen, dass sie mit den friberen Zellringen nichts zu thun haben.

Das Studium des Keimwalls bis zum Ende des ersten Tages erlaubt uns auch, die bisher gewonnenen Resultate zu controliren.

Ist es richtig, was wir bisher zu erweisen gesucht haben, dass der Keimwall ein Gemisch von Zellen und Dotterelementen, die vorwiegend in Umwandlung zu gelbem Dotter begriffen sind, repräsentire: ist es ferner begründet, dass die so wechselnde Gruppirung des Inhalts dieser letzteren manchmal Bilder vor Augen fuhrt, die entfernte Aehnlichkeit mit endogener Zellbildung haben: ist endlich aber wahr, dass es in keinem Falle zu einer Entstehung von Zellen in Dotterkugeln kommt, sondern dass alle Zellen im Keimwall ebensogut wie in der area pellucida, bereits vorhandenen Zellen ihre Entstehung verdanken: so muss ja die weitere Entwicklung die Beweise dafür beibringen. Sie muss zahlreiche Keimwallzellen, in Theilung begriffen, antreffen lassen; sie muss in allen verschiedenen Formzuständen der Dotterelemente das eine gemeinsame Moment enthalten, dass lediglich Auflösung, nicht Fortbildung daraus geschlossen werden kann. Wo sich Zellvermehrung findet, muss dieselbe an vorhandene Zellen sich anknüpfen lassen; und unter dieser Voraussetzung muissen die neu entstandenen Zellen sämmtlich, wie ihre Mutterzellen, in den Zwischenräumen der Dotterkugeln liegen, sie müssen frei, nicht etwa eingeschlossen sein. Es darf sich nur ihre Zahl, nnd die Art ihrer Vertheilung modificiren, nicht aber ihr morphologischer Charakter.

Ferner muss die zerstörende Wirkung der Erhärtungsfliussigkeit auf den Dotter des Keimwalls sich geltend machen; bald muss derselbe die Dotterkugeln erhalten zeigen, bald werden die meisten zerstört sein, so dass der Keimwall als eine formlose körnige 
Masse mit eingestreuten Zellkernen (da durch die Körner das Zellprotoplasma verdeckt wird) erscheint. Wir miissen also unter sonst gleichen Bedingungen einmal "zellige Gliederung “ des Keimwalls, ein anderes Mal keine Andeutung derselben erbalten; das Zellenmaterial dagegen muss immer in gleicher Weise erscheinen, da die Erhärtung es nur sehr wenig, und immer auf dieselbe Art verändert.

Dass nun dieses wirklich zutrifft, dass das constante Element die Zellen, das variable die Dotterkugeln des Keimwalls sind, erweist eine Vergleichung der Figg. 9, 10 und 13. Erstere ist ein Stuick der proximalen Zone eines 16 Stunden alten Keimwalls; Fig. 10 stellt eine entsprechende Partie nach 29stlundiger, Fig. 13 nach 34stündiger Bebruitung dar. In Fig. 9 und 10 zeigt sich keine Spur einer mosaikartigen Gliederung. Die ngrossen, grobkörnigen Zellen" sind vollständig verschwunden, und nur ihr körniger Inhalt als ungleichförmige, granulirte Masse erscheinend, ähnlich dem Boden der Keimhöhle, verdeckt zum Theil die Grenzen der eingestreuten Keimwallzellen. Geringer ist die Zerstörung der Dotterkugeln bei dem ältesten Keim. Man sieht eine grosse Anzahl völlig entleerter Kugeln (Fig. $13 \mathrm{Dk}$ ) und zwar an der inneren Grenze des Keimwalls; allmählich, je weiter man nach der Peripherie hin geht, treten Zellen zwischen diesen Kugeln auf. Auch sieht man viele nur zum Theil entleerte; endlich sind die Rindenschichten von einigen, dem Keim zunächst gelegenen nur zum Theil erhalten (Fig. $13 \mathrm{Dk}$ III). Es zeigt also dies Präparat zur Evidenz, einmal dass Keimzellen und Dotterkugeln im Keimwall nichts mit einander zu thun haben; ferner, dass die Dotterkugeln höchst variable Gebilde sind, endlich, dass ihr Inhalt sich unter Umständèn in Ballen gruppiren kann, die sehr entfernte Aehnlichkeit mit Zellen haben, aber dennoch bedeutend im Aussehen von denselben abweichen, auch gegen Carmin sich ganz anders verhalten.

Da ich früher $(10,1 . c$.) nachgewiesen habe, dass auf Dotterkugeln die Chromsäure diesen zerstörenden Einfluss ausüben kann, so nehme ich keinen Anstand, diese Entleerung der Dotterkugeln, sowie die Veränderung des Inhalts in den andern, auf Rechnung der Erhärtungsmethode zu setzen. Andererseits ist es ein Zufall, gerade solche Bilder zu treffen, die jede andere Deutung ausschliessen; übrigens zeigt ähnliche Dotterkugeln auch Fig. 20, woraus hervorgeht, dass dieser Zufall kein sehr seltener sein kann. 
Die Entstehung des Blutes und der ersten Gefässe im Hühnerei. 579

Obne ein eingehendes Studium der Reagenzwirkungen kann man die richtige Deutung derartiger Bilder schwerlich geben.

Der Dotter des Hühnereis hat eine Proteusnatur; seine mannigfachen Formen erinnern an Zellen und Zellaggregate; wären nicht charakteristische Momente da, welche mit Sicherheit eine Keimzelle von einer Dottermasse zu unterscheiden erlaubten, so die Beschaffenheit des Zellprotoplasma, die relative Grösse und das Lichtbrechungsvermögen des Zellkerns, das viel geringer ist als das der Dotterkörner, die immer vorhandenen, meistens doppelten Kernkörperchen, das Verhalten gegen Carmin, endlich die constant bleibende Grösse, was alles die Zellen hinlänglich characterisirt: würden nicht während der Bebritung die Unterschiede in so fern hervorstechender, als die Dotterelemente mannigfaltigere, aber stets regressive Umwandlungsprocesse durchmachen, während die einzige Veränderung der Zellen ihre Vermehrung durch Theilung ist: so wäre ein Auseinanderhalten beider Theile im Keimwall einfach unmöglich. So aber ist bei den Keimwallzellen die Formfolge eine constante, bei den Dotterelementen eine variable; die vorhandenen Unterschiede prägen sich durch die Entwicklung schärfer ans.

Es zeigt die Untersuchung des Keimwalls bis zum Ende des ersten Bebrütungstages zu allen Zeiten soviel in Theilung begriffene Zellen (Fig. 9, Z, Fig. 10), dass ich ihre Vermehrung allein auf diesen Vervielfältigungsmodus zurückführen muss.

Es wird also das im Keimwall vorhandene Zellmaterial erheblich vermehrt, aber es findet während des ersten Tages noch keine Verwendung. Der Keimwall bleibt eine zweischichtige Masse, deren obere Schichte das obere Keimblatt ist, deren untere Schichte, soweit sie aus Zellen besteht, als gleichwerthig dem mittleren und unteren Keimblatt gedeutet werden muss. Denn der Uebergang des Mesoblasten und Hypoblasten in den Keimwall bleibt wie in der Mitte des ersten Tages.

Es beginnt nun, wie His schon bestimmt angegeben hat, an der unteren Grenze des Keimwalls eine zusammenhängende Zellenlage aufzutreten. Aber dieselbe ist nicht uberall in gleicher Weise entwickelt; streckenweise findet sie sich, an andern Stellen wird sie vermisst, and es kommen auch unter ihr noch ab und zu Zellen im Dotter steckend, vor. An bestimmten Stellen ist freilich eine zusammenhängende Lage vorhanden; aber sie schliesst sich keinem 
der vorhandenen Keimblätter an. Ich möchte in ihrem Auftreten den Ausdruck der energiseben Zellvermehrung des Keimwalls sehen; wenn die Zellen immer zahlreicher werden, müssen die im gleichen Niveau liegenden schliesslich streckenweise zu Reihen zusammentreten.

Noch in den ersten Stunden des zweiten Tages fand ich den Keimwall so, wie bisher beschrieben; die Verwendung seines Zellmaterials beginnt nun um dieselbe Zeit. Es hatte die Zellvermehrung hauptsächlich in der proximalen Zone stattgefunden; und auf diese greift nun die Sonderung in Keimblätter über. Der Mesoblast tritt in der area opaca auf (Fig. 11).

Wie erfolgt die Ausdehnung des Mesoblasten?

Entweder durch peripherisches, centrifugales Wachsthum, das den Rand des Mesoblasten in die area opaca hinein schiebt, oder aber durch Anlagerung solcher Zellen, die vorher frei im Keimwall lagen. Wenn ersteres der Fall ist (centrifugales Wachsthum), so muss der Zellenreichthum des Keimwalls annähernd derselbe bleiben; wenn aber der Zellreichthum des Keimwalls abnimmt, so liegt am nächsten eine Verschiebung seiner Zellen anzunehmen, und auf diesen Vorgang, dem eine Anlagerung der dislocirten Zellen an die untere Fläche des Epiblasten folgt, die Bildung einer zusammenhängenden Lage unter diesem zurückzuführen. Tritt dann, diese Zellenschichte mit dem Mesoblasten der area pellucida in Verbindung, so kann man sagen, dass der Mesoblast anf die area opaca ïbergreife.

Nun finden wir im Anfang des zweiten Tages einen sehr zellenreichen Keimwall (Fig. 10), eine geringe Zeit nachher finden wir den Keimwall zellenarm und auf ihm, dicht unter dem Epiblasten, eine Lage von wechselnder Dicke, aus Rundzellen zusammengesetzt, die mit dem Mesoblasten der area pelluicida nicht in Verbindung steht (Fig. $11 \mathrm{~A}$, $B, C)$. Soweit diese Zellenlage reicht und noch etwas weiter, ist der Keimwall zellenarm (Fig. $11 \mathrm{~K} \mathrm{w}$ ).

Dass die fragliche Zellenschichte der Mesoblast sei, ist unzweifelhaft; ebenso unzweifelhaft ist aber zu sehen, dass sie noch nicht mit dem Mesoblasten der area pellucida in Verbindung steht. Sie muss also in loco entstanden sein, und dazu war Material genug vorhanden. Wenn eine fruher zellenreiche Dotterschichte in einem späteren Stadium sehr zellenarm erscheint, wenn gleichzeitig an 
Die Entstehung des Blutes und der ersten Gefässe im Hühnerei. 581

ihrer oberen Grenze eine Zellenlage auftritt, die im frtiheren Stadium nicht da war, und wenn diese Zellenlage keine Verbindung mit einer anderen hat, so muss man ihre Bildung auf Verlagerung der früher im Dotter steckenden Zellen zurïckführen. Es bleibt keine andere Deutung ubrig.

Das Auftreten des Mesoblasten in der area opaca ist also ein durchaus selbständiges; er bildet sich durch Concentrirung der Keimwallzellen und Anlagerung derselben an die untere Fläche des Epiblasten. So entsteht eine unregelmässig dicke, aus dickeren, kugligen, schmäleren, platten Abtheilungen zusammengesetzte Zellenlage, welche derart dem Keimwall aufliegt, dass die Verdickungen in entsprechende Vertiefungen des Keimwalls eingreifen (Fig. 14, Fig. $15 \mathrm{M} \mathrm{s} \mathrm{b).}$

Dabei wird der Zellreichthum des Keimwalls erheblich reducirt, aber es bleiben noch Zellen im selben zurück (Fig. 11, 14, 15).

Die Mesoblastbildung im Keimwall schreitet in der Richtung von hinten nach vorn vor, da wo das Rückenrohr zuerst sich schliesst, ist die vordere Grenze. Hinter dieser Stelle reicht der Mesoblast in die area opaca hinein, vor ihr treffen wir den Keimwall so, wie er im Verlauf des ersten Tages war.

Die nach oben verlagerten Keimwallzellen treten sämmtlich zum Mesoblasten, und es tritt der Mesoblast der area pellucida mit dem der area opaca sehr bald in Verbindung. Die Verbindungsstelle kennzeichnet sich dadurch, dass sie schmaler ist als die übrigen Mesoblastpartien. Der Hypoblast greift nicht in die area opaca über; er hört an der inneren Grenze des Keimwalls mit leicht verdicktem Rande auf (Fig. 11, $\mathrm{Hpbl}$ ).

Ich sagte schon oben, dass der Mesoblast in der area opaca unregelmässig dick sei (Fig. 11); das legt die Vermuthung nahe, dass die Keimwallzellen zunächst im Keimwall zu Haufen sich concentriren, dass diese Haufen nach oben gerückt sind, dem Epiblasten sich angelegt haben, und dass eine Verbindung der Zellhaufen durch Zellen sich erst nachträglich vollzogen hat. Nun habe ich einen Zellhaufen, noch im Keimwall steckend, bei einem auf dem Stadium der Fig. 11 stehenden Keim wirklich angetroffen (Fig. $15 \mathrm{~b}$ ), derselbe steht durch eine etwas lückenhafte Zellenreihe mit dem Mesoblasten auf dem Keimwall in Verbindung.

Die Bildung von Zellenhaufen im Keimwall geht daraus her- 
vor, und da der Zellenhaufen augenscheinlich dem Mesoblasten sich anzulagern im Begriff steht, also der Mesoblast in der area opaca durch Apposition wächst, so kann wohl die Ansicht motivirt erscheinen, dass er itberhaupt nach dem Typus angelegt sei, nach welchem er sich vergrössert. Er vergrössert sich nun aber nicht allein durch Anlagerung von Zellhaufen, sondern auch von einzelnen Zellen (Fig. 14 a b). Dieselben kommen aus dem Dotter des Keimwalls nach oben, und legen sich der unteren Fläche des Mesoblasten an; daher erscheint diese unregelmässig, und ist durchaus nicht scharf rom Dotter abgesetzt.

Sehr bald nach seiner Bildung tritt der Mesoblast der area opaca in Verbindung mit dem der area pellucida. Er wächst immer weiter in die proximale Zone hinein, und vergrössert sich, wie wir gesehen haben, durch appositionelles Wachsthum. Da der Hypoblast nach wie vor in den Keimwall sich verliert, so kommt natürlich die Mesoblastverbreiterung auf den Keimwall zu liegen.

Die Zellhaufen im Mesoblasten der area opaca erscheinen nun im Flächenbilde als cylindrische oder unregelmässig begrenzte Massen; sie sind die ersten Anlagen sowohl des Blutes als der Gefässe.

Sie finden sich nur im mittleren Keimblatt, und nur in der area opaca. Die Zeit ihrer Entstehung ist die erste Hälfte und die Mitte des zweiten Tages; immer sind sie da, bevor die Spaltung des Mesoblasten in Haut- und Darmfaserplatte, also die Bildung der Pleuroperitonealhöhle, beginnt. Hat diese Spaltung begonnen, so sondert sich auch der Mesoblast der area opaca; es wird dann deutlich, was zu Blut, was zu Gefässwand wird.

Wenn in der area pellucida die Bildung der Pleuroperitonealhöhle erfolgt ist, greift die Spaltung des Mesoblasten in Hautfaserplatte und Darmfaserplatte auf die area opaca über. Erstere legt sich hier dem Epiblasten dicht an; letztere dagegen zieht, parallel dem Epiblasten, frei tiber den Keimwall hin (Fig. 12, D f p; Fig. 17, D f p). Beide Platten sind leicht zu erkennen, da sie sich in die area pellucida hinein verfolgen lassen; beide sind nur einfache Lagen, und die sie bildenden Zellen nähern sich der Spindelform, während vor Bildung der Pleuroperitonealhöhle der Mesoblast in der area opaca ausschliesslich Rundzellen zeigte. Es bleiben aber ausser beiden Platten noch Zellen in der area opaca uibrig, die zum Mesoblasten gehören, aber keiner der erwähnten Lagen 
Die Entstehung des Blutes und der ersten Gefässe im Hühnerei. 583

sich anschliessen; es sind dies Haufen von Rundzellen und zwischen ihnen finden sich streckenweise Reihen ron Spindelzellen vor. Diese bleiben unter der Darmfaserplatte liegen (Fig. 12, Fig. 17, G f p Bl.).

Sie berühren also den Dotter des Keimwalls und heben da, wo sie sich finden, die Darmfaserplatte vom Keimwall ab. Es findet sich demnach in der area opaca ausser Haut- und Darmfaserplatte noch eine dritte, aus dem Mesoblasten abgespaltene Zellenschicht, von wechselnder Mächtigkeit; man kann dieselbe eben so gut als selbständige Platte ansehen, als die beiden ersteren. $\mathrm{His}$, der ihre Bildung näher beschrieben hat, aber sie aus dem weissen Dotter des Keimwalls ableitet, hat sie als „Gefässblatt" bezeichnet; ich will sie, um ihre Gleichwerthigkeit mit den beiden andern, aus dem Mesoblasten abgespaltenen Lagen hervorzuheben, lieber "Gefässplatte" nennen. Sie begreift alle Zellen und Zellenanhäufungen in sich, die unter der Darmfaserplatte gelegen sind (Fig. 12, Fig. 17, Gfp).

Ist sie also schon durch die Lage gekennzeichnet, so weicht sie auch im Bau einigermassen ab. Sie ist keine ununterbrochene Membran, wie die Darmfaserplatte, sondern man trifft sie nur streckenweise auf Durchschnitten an (Fig. 12, Gfp) sie hat also L ỉ cken. Ausserdem findet sie sich anfangs nur in der area opaca; in die area pellucida gelangt die Gefässplatte kurze Zeit später hinein.

Wo die Gefässplatte vorhanden ist, liegt sie unmittelbar dem Keimwall auf; wenn also noch nach ihrer Bildung Zellen ans dem Keimwall zum Mesoblasten gelangen, so treten sie an die Gefässplatte, oder in eine Lücke derselben hinein.

Die Rundzellenhaufen sind nun bald spindelförmigeVerdickangen der Gefässplatte (His), bald aber liegen sie derart, dass die Darmfaserplatte sie an ihrer oberen Seite begrenzt, während eine zweite Spindelzellenlage sie vom Dotter trennt, und zu beiden Seiten des Zellhaufens mit der Darmfaserplatte verwächst. (Fig. 12a r, a II, a III, Fig. 17). Die Rundzellenhaufen kommen dadurch in Hohlräume zu liegen, die oben von der Darmfaserplatte, unten von den Spindelzellen der Gefässplatte geschlossen werden; dass aber die untere Spindelzellenschicht nicht einfach aus veränderten Rundzellen der Peripherie hervorgegangen ist, wird dadurch bewiesen, dass in der area opaca und besonders in der area pellucida derartige Verwachsungen von Gefässplatte und Darmfaserplatte sich finden, 
ohne dass ein Rundzellenhaufen eingeschlossen wäre (Fig. $12 \mathrm{G}$ ). Ich folgere daraus, dass die Spindelzellen unter der Darmfaserplatte auch da vorkommen, wo keine Anhäufungen von Rundzellen liegen, und das bestimmt mich, sie als Theile einer eigenen Platte anzusehen und zu benennen.

Fig. 12 sowohl wie Fig. 17 sind aber Durchschnitte von Partien, in denen schon Gefässe, theils gefüllt, theils leer, sich finden; es folgt zeitlich das in Fig. 12 fixirte Stadinm anf das in Fig. 14 wiedergegebene. Wie die Bildung der ersten Blutbahn erfolgt, ist in diesem Stadium deutlich $z u$ sehen.

Die Gefässe legen sich an als Spalträume, die durch Verwachsung von Gefässplatte und Darmfaserplatte entstehen. Wo ein Rundzellenhaufen im Mesoblasten lag, bleibt er bei dem Zerfall desselben in die 3 Platten unberührt vomZerfall liegen und darauf gelangt er in die durch Verwachsung der beiden untersten Platten entstehenden Spalträume (Fig. 12, Fig. 17 Bl).

Auf den Modus dieser Verwachsung miissen wir noch näher eingehen. Schon oben ist gesagt, dass die Gefässplatte L ti cken habe; darum trifft man sie auf dem Durchschnitt bald an, bald wieder nicht. Wo die Gefässplatte auf dem Durchschnitt nicht zu finden ist, und die Darmfaserplatte dem Keimwall aufliegt, ist eine solche Lucke; und jedesmal an den Rändern eines derartigen Loches ist die Gefässplatte mit der Darmfaserplatte verwachsen (Fig. 12, ar, a Ir ).

Der Durchschnitt kann aber die Weite der Luicken besser zeigen, als ihre Form. Diese erscheint nur in der Flächenansicht. Nehmen wir vorläufig einmal an, alle Löcher der Gefässplatte seien gleich gross, und besässen die Form von Kreisen. Dann können wir, allein gestiitzt auf das Studium von Durchschnitten, uns schon eine Vorstellung von dem Verhältniss beider Membranen, der Gefässplatte und der Darmfaserplatte machen, die im wesentlichen richtig ist und uns das Verständniss der Flächenbilder ausserordentlich erleichtern muss. Es ist nämlich dann die Darmfaserplatte zu vergleichen einem Bogen Papier, dem ein ebenso grosser, aber überall von kreisförmigen gleich grossen Oeffunngen durchbohrter Bogen untergelegt ist. Nun decken wir beide Papierstiicke genau aufeinander, verkleben sie mit einander an ihren Rändern und an den Rändern der Löcher, die dem unteren eingeschlagen sind. Dann muss zwischen beiden ein System gleich weiter, anastomosirender 
Die Entstehung des Blutes und der ersten Gefässe im Hühnerei. 585

Canäle entstehen; die Maschen dieses Netzes von Gängen mùssen kreisförmig sein, und genau so gross wie die Löcher des unteren Papierstückes; selbstverständlich sind sie identisch mit diesen.

Legt man nun beide, in der angegebenen Art verbundene Bogen auf eine dunkle Unterlage, so scheint diese in den Maschen des Canalnetzes durch; die Interstitien zwischen den Canälen erscheinen dunkel, die Canäle hell. Die Grenzen der dunkeln „Substanzinseln" sind aber, als Verklebungsstellen beider Platten, do ppelt so dick als die obere Platte allein; sie sind kreisförmige Verdickungen der oberen Platte, und bilden also verdickte Ringe um die "Substanzinseln“.

Genau wie das Verhältniss dieser beiden Papierstlicke ist nun das Verhältniss von:Darmfaserplatte und Gefässplatte. Einzig die Form der Löcher ist geändert. Die Unterlage für beide ist der Dotter des Keimwalls. Derselbe füllt scheinbar die ringförmigen Verwachsungsstellen beider Membranen aus; er wird, anders ausgedrückt, an jeder Verwachsungsstelle von einer ringförmigen oder länglichen Verdickung der Darmfaserplatte umfasst (Fig. 16 A).

Kann man nun aber erwarten, bei der Flächenansicht eines ganzen Keims die beiden Zellenlagen zu seben, welche die Darmfaserplatte bedecken? Wenn man Osmiumpräparate anwendet, nieht. Denn Osmium färbt den Dotter des Keimwalls stark, die Zellen des Epiblasten und die Platten des Mesoblasten dagegen fast gar nicht, wenigstens nicht, wenn man ganz dünne Lösungen (ich wendete eine Lösung von $1 / 10 \%$ an) nur kurze Zeit einwirken lässt. Den Epiblasten und die Hautfaserplatte sieht man ron oben her ebensowenig wie die Dotterhaut, weil beide ganz diunne, durchsichtige, parallele Membranen sind, deren Dicke im Verhältniss zu der des gefärbten Keimwalls sehr zurïcktritt (vgl. den Durchschnitt Fig. 12).

Man sieht auch mit starken Systemen (und meine Präparate erlauben die Anwendung von Winkel's System 8 noch ganz bequem) nur die Zellenhaufen auf dem Keimwall (Fig. 18, Fig. 19 Bl) and die verdickten Stellen der Darmfaserplatte, also die Zellenringe, in denen der Dotter des Keimwalls zu liegen scheint, die "Substanzinseln" der Autoren. Gleichfalls sieht man das Canalsystem zwischen Gefäss- und Darmfaserplatte; dasselbe erscheint zwischen ringförmigen Substanzinseln ausgespart (Fig. 19). Dotterhaut, Epiblast und Hautfaserplatte sind in der area opaca 
uberall gleich dick; sie brechen das durchfallende Licht so unbedeutend, dass sie nicht zu sehen sind.

Die Zellenringe erstrecken sich aber viel weiter als die Blutinseln (Fig. 18, Fig. 19); sie füllen schon zu einer Zeit die hintere Hälfte der area pellucida, in der erst vereinzelte Blutkörperchen in den Embryonaltheil hinein gelangt sind. Die Gefässplatte also breitet sich viel rascher in die area pellucida aus, als die strangförmigen und kugligen Blutmassen; die Räume, in denen das Blut in den Embryo hineinkommt, legen sich zuerst an, und durch praeformirte Bahnen bezieht der Embryo das in der innern Zone der area opaca gebildete Blut. Auch Durchschnitte zeigen leere Gefässlumina in der area pellucida viel früher, als gefüllte auftreten. Sie stimmen also mit den Flächenbildern iuberein und man muss ein unabhängig von den Zellenhaufen der area opaca vor sich gehendes Wachsthum der Gefässplatte annehmen.

Wiederholt habe ich erwähnt, dass auch nach dem Auftreten der Mesoblasten in der area opaca im Keimwall noch Zellen anzutreffen sind (Fig. 12, Fig. 14, Fig. 15). Diese legen sich, wenn sie nahe der oberen Grenze des Dotters angekommen sind, der Gefässplatte an; hat diese ein Loch, so treten sie bis an die Darmfaserplatte. Von der Fläche betrachtet, liegen sie dann innerhalb der Zellenringe, innerhalb der verdickten Partien der Darmfaserplatte (Fig. $19 \mathrm{~S} \mathrm{i)} \mathrm{und} \mathrm{sie} \mathrm{können} \mathrm{bald} \mathrm{näher,} \mathrm{bald} \mathrm{entfernter} \mathrm{von}$ der Wand der Substanzinsel angetroffen werden. Wer nun nicht weiss, wie der Zellenring entsteht, kann auf die Idee kommen, es habe sich die im Ring liegende Zelle von seiner Wand abgeschntirt. Dann würde eine zellenhaltige Substanzinsel einer Klein'schen Endothelblase identisch sein; und ich vermuthe, dass die ganze Theorie von Klein auf einer derartigen Deutung der Flächenbilder beruht. Nur müsste, nach Klein's Auffassung, das Blut innerhalb der Zellenringe liegen, während jedes einigermassen brauchbare Flächenbild das Blut in Bahnen zeigt, die $\mathrm{zw}$ is chen benachbarten Ringen liegen. Diese Thatsache allein würde schon genẗgen, die ganze Deutung von Klein als unhaltbar erscheinen zu lassen.

Richtiger hat Afanasieff die Zellringe des Flächenbildes aufgefasst; er erkannte, dass das Blut $\mathrm{zw}$ is chen ihnen, nicht in ihnen liegt. Da er aber die Bedeutung der Ringe, ihre Entstehungsweise, nicht erkannte, sah er sich in die Nothwendigkeit versetzt, von der Wand derselben die Blutkörperchen bilden zu lassen; er musste 
aber eine Abschnürung derselben nach Aussen, nicht nach Innen, statuiren. Vielleicht hat Afanasieff, der vorwiegend die area pellucida untersuchte, die ersten hineingelangenden Blutzellen der Gefässwand zunächst liegend angetroffen, und diesen Befund für seine Deutung verwerthet.

Nach dem Vorstehenden muss ich also die Angabe Kölliker's, dass das erste Blut in Form solider Zellenstränge im Randtheil des Mesoderma auftrete, in ihrem vollen Umfange bestätigen.

Nur über die Herkunft des peripheren Theils des Mesoblasten besteht eine untergeordnete Differenz zwischen Kölliker's and meinen Angaben. Kölliker leitet denselben ab aus dem Mesoblasten der area pellucida, während ich, gestiitzt auf Fig. 6, 7, 9, 10, 11, sein Auftreten auf die Zellen des Keimwalls zurückführe. Da ich aber dieselben ebenso auf Furchungszellen zurückführeñ muss, wie die ubrigen Zellen des Keims, so erscheint mir diese Differenz, ob es sich um centrifugales oder um appositionelles Wachsthum des Mesoblasten handelt, nicht als eine principielle.

Ferner muss ich Kölliker's Angaben bestätigen, dass zuerst solide Zellstränge da sind, und dass nals zweites Stadium Hohlgebilde auftreten". Den Modus der Hohlraumbildung berïhrt Kölliker nicht näher. Gestützt auf Combination von Durchschnitten und Flächenansichten, halte ich es für gerechtfertigt, eine "Gefässplatte" aufzustellen und anzunehmen, dass dieselbe durch multiple Verwachsung mit der Darmfaserplatte die ersten Blutbahnen bilde. Vgl. Fig. 12, Fig. 17. Ohne die Annahme eines selbständigen Wachsthums dieser Platte in den Embryo hinein lassen sich die Flächenbilder nicht in befriedigender Weise deuten, denn es bleibt dann die Regelmässigkeit der Bildung, und die alleinige Sichtbarkeit der „Substanzinseln“ unerklärlich. Dass unter der Darmfaserplatte und zwar zuerst in der area opaca, noch Zellen liegen bleiben, zeigt jeder Durchschnitt. Fassen wir diese in ihrer Gesammtheit als dritte Platte des Mesoblasten, so lässt sich nichts dagegen einwenden, wenn wir dadurch uns das Verständniss gewisser Vorgänge erleichtern. Und das ist der Fall. Sicher ist, dass die Darmfaserplatte stets an der Bildung der ersten Gefässwand sich betheiligt (Fig. 12, Fig. 17), sie bildet die obere, die Gefässplatte die untere Wand. Gestützt anf diese Thatsache wird uns die Betheiligung der Darmfaserplatte an der Bildung der Herzwand klar. 
Das Herz ist eines der vordersten, und eines der der Mittellinie nächsten Gefässe. Aus beiden Grïnden legt es sich erst später an, als die meisten tibrigen Blutbahnen, die ja von hinten und aussen nach vorne und innen vorschreiten. Es betheiligt sich nun an der Herzbildung die Gefässplatte und die Darmfaserplatte. Diese letztere bildet aber jederseits von der Medianlinie eine Falte, deren Concavität gegen den Hypoblasten gekehrt ist. Die Gefäissplatte macht diese Falte mit; sie läuft der Darmfaserplatte parallel, und kommt dadurch, dass die Falte sich zu einem Ringe schliesst, in das Innere des Ringes hinein zu liegen. (Vgl. Kölliker, 9, Fig. 208, 209, pag. 290.)

Die Gefässplatte bildet beim Herzen das Endothe1rohr, während dieDarmfaserplatte die Musculaturliefert.

Dass auch bei den übrigen Gefässen dieGefässplatte, die anfangs einen Halbring bildet (vgl. Fig. 12) sich zu einem rollständigen Ringe schliesst und zum Endothelrohr wird, ist mir in hohem Grade wahrscheinlich; denn ich habe an Embryonen vom Anfange des 3. Tages in der area pellucida vollkommen geschlossene Ringe von Spindelzellen zwischen Darmfaserplatte und Hypoblasten angetroffen, also offenbar Gefässquerschnitte, die weiter entwickelt waren als die in Fig. 12 und 17. Beim Herzen trennt sich die Gefässplatte von der Darmfaserplatte und schliesst sich zum Ringe; warum soll es bei den übrigen Gefässen nicht auch so sein? Für die Bildung der Muscularis könnte ja immer die Darmfaserplatte sorgen, wie sie ja bei dem Gefässe mit der stärksten Musculatur, dem Herzen, dasselbe nachweisbar thut, und wie sie auch das Darmepithel mit contractiler Substanz versorgt. Dass die Darmfaserplatte sowohl glatte als quergestreifte Musculatur bilden kann, scheint mir doch die Untersuchungen über ihre Betheiligung an der Entwickelung des Herzens zweifellos erwiesen; keinesfalls lässt sich eine Trennung glatter und quergestreifter Muskeln in morphologischer Hinsicht aufrecht erhalten, da - ich erinnere an die Musculatur der Mollusken - massenhaft Uebergänge zwischen beiden Formen sich finden.

Darauf machte mich schon vor längerer Zeit mein verehrter Freund, Herr Dr. v. Iher ing aufmerksam; der Sehliessmuskel der Muscheln z. B. ist ein geeignetes Untersuchungsobject (vgl. v. Ihering, Ueber Anomia etc. Zeitschrift f. wissenschaftl. Zoologie Bd. XXX Suppl. pag. 26). 
Da ich nur die erste Entstehung der Gefässe behandeln will, nicht aber ihre weitere Entwicklung, so will ich hier nicht weiter auf die Bildung des Endothelrohrs und der Muscularis eingehen.

Es erstreckt sich der Mesoblast nur über einen Theil der proximalen Zone des Keimwalls. Der äussere Theil desselben bleibt, wie er am Ende des ersten Tages war. Auch im Keimwall unter dem Mesoblasten bleiben, wie erwähnt, Zellen zurtick. Dieselben gelangen allmählig in die oberen Parteien desselben, und einzelne treten dicht an die untere Fläche der Gefässplatte heran. Indem nun um diese Zeit, gegen Ende des zweiten Tages, der Keimwall bedeutend an Mächtigkeit abnimmt, was ich auf Resorption seines Dotters zurlickfüren möchte, bleibt der Keimwall an den meisten Stellen nicht dicker, als der Durchmesser einer Dotterkngel beträgt. Die Dotterkugeln selbst platten sich an einander ab und erscheinen wie hochcylindrische Zellen, deren Kerne in den Fuss, d. h. den der Gefässplatte zugewandten Theil der Zelle geriuckt sind. H. Virchow und Kölliker haben die Kerne der Keimwallzellen als Kerne der Dotterkugeln gedeutet und angegeben, dass der Keimwulst schliesslich zu einer einschichtigen Zellenlage werde. Man erhält dieses Bild, wenn der körnige Inhalt der Dotterkugeln derart die Zellgrenzen verdeckt, dass man nur die Kerne der letzteren sieht.

Fassen wir die Resultate vorstehender Untersuchung kurz zusammen, so ergiebt sich folgendes. Aus dem Randwulst des unbebrïteten Eies geht der aus Zellen und Dotterelementen gemischte Keimwall hervor (Fig. 2, 4, 6, 7). Die Zellen desselben vermebren sich im Laufe des ersten Tages bedeutend, theilen sich aber vorerst noch keinem Keimblatt zu (Fig. 9, 10). Im Anfange des zweiten Tages concentriren sich die Keimwallzellen zu Haufen (Fig. 15), treten unter den Epiblasten und bilden eine unregelmässig dicke Zellenlage, die mit dem Mesoblasten der area pellucida in Verbindung tritt (Figg. 11, 14). In dieser peripheren $\mathrm{Me}-$ soblastpartie entsteht durch Sonderung einer gemeinsamen Anlage sowohl Blut, als Blutbahn; die Blutentstehung bleibt auf diese 
Partie des Mesoblasten beschränkt, die Gefässbildnng schreitet, unabhängig von den Blutmassen, in die area pellucida hinein vor, und die ersten Gefässe entstehen durch vielfache Verwachsungen zwischen Darmfaserplatte und Gefässplatte. Das Herz legt sich nach demselben Princip an (Fig. 12, 17, 18, 19, 16).

Wenn also auch das Material, dem das Blut entstammt, vorwiegend im Keimwall sich ansammelt; es entsteht weder aus Dotterelementen des Keimwalls, wie $\mathrm{H}$ is will, noch aus Furchungszellen, die von der Keimhöhle her in den Keimwall eingewandert sind, wie Goette angegeben hat. Auch tritt nicht im Keimwall, sondern a u dem Keimwall, im peripheren Theil des Mesoblasten die Blut- und Gefässbildung ein; es entstehen nur im mittleren Keimblatt, keineswegs uuabhängig von demselben, die Blutkörperchen und die ersten Gefässe.

Die Angaben von Remak und Kölliker sind also in allen wesentlichen Punkten begründet; die spüteren Angaben, welche in Hauptpunkten von den Resultaten dieser Forseher abweichen, beruhen nach meinen Erfahrungen sämmtlich auf mangelhaften Deutungen der Flächenbilder, wie die von Afanasieff, Klein und Balfour, oder auf ungenugender Trennung dessen, was Dotter ist und was Zellen, wie die Angaben von $\mathrm{Hi}$ is und Goette.

Weshalb ich aber den „Keimwulst" Kölliker's nicht anerkennen kann, ist hinlänglich aus meinen bezüg]ichen Ausführungen zu ersehen. Der Keimwulst ist ein Gemisch ron Zellen und Dotter, keineswegs besteht er aus Zellen allein; seine Zellen betheiligen sich vorwiegend an der Bildung desjenigen Theils des Mesoblasten, in dem das erste Blut auftritt. Dies ist der einzige Punkt, in dem meine Angaben mit denen Kölliker's in Widerspruch stehen; wie gesagt, halte ich die Differenz für nicht sehr wichtig, da sie nur die Art, wie der Mesoblast wächst, betrifft. Das Zellmaterial, das ihn nach meiner Auffassung bildet, stammt ja ebenso von Keimzellen $a b$, wie die übrigen Zellen des Mesoblasten, und die Einheit der Anlage aller Gewebe des Organismus bleibt gewahrt.

$\mathrm{Zu}$ erklären bleibt indess noch, wie es kommt, dass die Dot- 
Die Entstehung des Blutes und der ersten Gefässe im Hühnerei. 591

terkugeln im Keimwall sich zu einer Lage gruppiren, die meistens eine directe Fortsetzung des Hypoblasten zu sein scheint: wie es kommt, dass unter der area pellucida meistens die Dotterkugeln zerstört werden, während sie in der area opaca in den meisten Fällen erhalten bleiben, so dass der Keimwall auf Durchschnitten sowie auf Flächenschnitten ein mosaikartiges Aussehen zeigt. Es ist freilich wahr, dass der Keimwall keineswegs ein so constantes, in allen Fällen unveränderliches Bild liefert, wie etwa die aus Zellen bestehenden Keimpartien, z. B. der Epiblast; es kommt vor, dass das zellige Aussehen des Keimwalls ganz verschwindet, oder auch nur andeutungsweise hervortritt. Ich vermuthe, dass im Keimwall die resistentesten Dotterkugeln vorhanden sind, so dass bei der Erhärtung nur ein kleiner Bruchtheil jedesmal zerstört wird; ich kann aber nicht erklären, warum hier die granulirten $\mathrm{Kug}$ eln resistenter bleiben als anderswo.

Dass es sich aber keineswegs um Zellen handelt, geht wohl zur Genüge aus meiner Darstellung hervor.

Die Untersuchungsmethoden waren dieselben, wie ich sie friber angewandt und mitgetheilt habe (10, pag. 78,79$)$. Für Flächenpräparate benutzte ich schwache Osmiumlösung, 1/10\%, die ich 10-15 Minuten einwirken liess. Den Keimwall zeigen nur Präparate, die mit Carmin oder $\mathrm{Picrocar}$ min gefärbt sind; an Osmi umpräparaten sieht man die hell bleibenden Zellen im dunkeln Dotter durchaus nicht. Carmin färbt eben nur die Zellen, und lässt den Dotter absolut intact. Haematoxylin färbt den Dotter tiefblau, die Zellen schwächer, und ist daher zur Darstellung des Keimwalls ungeeignet.

Wie schon herrorgehoben, lag es nicht in meiner Absicht, die Untersuchung auf die weitere Ausbreitung der primären Blutbahnen im Embryo und die Bildung der secundären Gefässe auszudehnen. Nach den bis jetzt daruber vorliegenden Untersuchungen scheint allerdings eine andere Bildungsweise der letzteren stattzufinden, sowohl bei der pathologischen Neubildung, als bei der Vascularisation von Theilen in späteren Stadien des foetalen Lebens. Die Capillaren wenigstens entstehen durch Hohlwerden von Zellen, welche benachbarte Gefässe mit einander verbinden; ob nicht der- 
artiges kurz nach Bildung der primären Blutbahn im Bereich der area pellucida $a b$ und $z a$ vorkomme, will ich nicht direct in Abrede stellen. Balfour scheint solche Vorgänge beobachtet $z \mathfrak{u}$ haben; sie wïrden aber schon in den Bereich der secundären Gefässbildung fallen und höchstens beweisen, dass diese zn sehr fruher Zeit schon beginnt.

Ebenso ist die Frage, ob nicht später Blutkörper unter Betheiligung der Gefässwand sich bilden, noch einer genaueren Prífung werth. Nach meinen Untersuchungen entstehen die ersten Blutmassen unabhängig von den ersten Gefässwänden; aber angesichts der Untersuchungen von Wis sozky will ich meine Resultate nicht generalisiren, und für alle im Laufe der embryonalen Entwicklung weiter gebildeten Blutkörper als gültig hinstellen. Was die Bildung der secundären Gefässe anbetrifft, so muss ich sagen, dass eine erschöpfende Bearbeitung aller einschlägigen Verhältnisse, während des Embryonallebens und des spätern Wachsthums, bei der Neubildung von Geschwtilsten und bei der Wundheilung, erst die Anhaltspunkte liefern kann, um die Entstehungsweise derselben klar zu legen; ich enthalte mich daher eines jeden Urtbeils darüber, muss aber hervorheben, dass der Nachweis, die secundären Gefässe entständen z. B. sümmtlich durch Hohlwerden von Zellen, durchaus nicht als Argument gegen die Angaben dienen könnte, dass die primären Blutbahnen als Intercellularräume sich anlegen. Es würde nur daraus hervorgehen, dass die späteren Gefässe anders sich bilden als die ersten; und dafür sprechen auch die vorliegenden Untersuchungen ron Ranvier und Wissozky. (Vgl. Ranvier Archives de Physiologie par Brown-Séquard, 1874. pag. 428; Wissozky, Archiv für mikrosk. Anatomie, Bd. XIII. pag. 479.) 


\section{Erklärung der Abbildungen auf Tafel XXVI-XXVIII.}

Fig. 1. Keim v. 24 Stunden, Osmium-Chromsäure. Querschnitt des hinteren Endes des Primitivstreifs. Prst Primitivstreif, eine axiale Verdickung des Mesoblasten, der mit dem Hypoblasten $\mathrm{Hpbl}$ untrennbar verbunden ist. Hartnak III/4; eingeschobener Tubus.

Fig. 2. Derselbe Keim, 9 Schnitte weiter vorn. Verwachsung des Epiblasten mit dem Mesoblasten im Bereich der Primitivrinne. E pb. Epiblast; Ms b Mesoblast, Hpbl Hypoblast. Prn Primitivrinne. Hartnak $\mathrm{III} / 4$, eingeschobener Tubus.

Fig. 3 (identisch mit Fig. 5 meiner früheren Arbeit). Unbebrüteter Keim; Trennung von Randwulst und Keimwall. 0 s obere Keimschicht; $\mathrm{r}$ w Randwulst der unteren Schichte; $\mathrm{K}$ w Keimwall. KI, K II granulirte Dotterkugeln im Randwulst. Leitz I/5, eingeschlobener Tubus.

Fig. 4 (identisch mit Fig. 7 meiner früheren Arbeit). Keim, 9 Stunden bebrütet. Erste Anlage des Mesoblasten, Uebergehen des Randwulstes in den Keimwall. Ep b Epiblast; p r Primitivstreif, Mesoblast, $\mathrm{hpb}$, Hypoblast. $\mathrm{K} \mathrm{w}$ Keimwall, d $\mathrm{z}, \mathrm{pzn}$, distale und proximale Zone desselben. a, b, Uebergang des Hypoblasten in den Keimwall. Leitz $I / 5$, eingeschobener Tubus.

Fig. 5 Keim v. 11 Stunden. Schnitt durch die area opaca, senkrecht auf den Primitivstreifen, hinter dem Schwanzende des Embryo. Zusammensetzung des Keimwalls aus Dotter und Zellen. E p b Epiblast, $\mathrm{K}$ w Keimwall, entsprechend dem Mesoblasten und Hypoblasten zusammen. A distale Zone, bei stärkerer Vergrösserung dargestellt in Fig. 6; $B$ das in Fig. 7 gezeichnete Stück der proximalen Zone. Winkel II/2.

Fig. 6. Distale Zone des Keimwalls. Z. Keimwallzelle mit grossem Kern und dünnem, aber deutlich sichtbarem Zellprotoplasma; $\mathrm{Dk}_{1}$ Dotterkugel mit feingranulirtem Inhalt. D k II Dotterkugeln, die einen Zellkern zu umschliessen scheinen, da ihr Inhalt die Zellgrenze verdeckt. Dk $\amalg$ kleinere Dotterkugeln. Epb Epiblast. Winkel II/8.

Fig. 7. Stïck der proximalen Zone des Keimwalls. Ep b' Epiblast; Z Zellen mit deutlichem Protoplasma ähnlich den Epiblastzellen. D kı Dotterkugeln. Winkel $\mathrm{I} / 8$.

Fig. 8. Derselbe Keim; Uebergang der beiden untern Keimblätter in den Keimwall; mittleres und unteres Keimblatt noch nicht deutlich getrennt. A Grenze zwischen area pellucida und area opaca, innere Grenze des Keimwalls. D k granulirte Dotterkugeln, eine davon mit 
einem scheinbaren Kern. Epb Epiblast, Kw Keimwall, mit vielen Dotterkugeln und Zellen dazwischen. Z Keimwallzellen. Winkel II/8.

Fig. 9. Keim v. 16 Stunden. Stück der proximalen Zone des Keimwalls. Theilung der Keimwallzellen; die Dotterkugeln im Keimwall meistens zerstört. Epb Epiblast. $Z_{\text {I }}$ Keimwallzellen in Theilung. G D Gelber Dotter an der unteren Grenze des Keimwalls. Winkel II/8.

Fig. 10. Keimwall eines Eis von 29 Stunden. Bedeutender Zellenreichthum der proximalen Zone; gruppenweise Anordnung der Keimwallzellen. Der Epiblast ist weggelassen. GD gelbe Dotterkugeln an der unteren Grenze des Keimwalls, $Z$ sternförmige Zelle zwischen denselben frei liegend. $\mathrm{Z} G$ Zellengruppen; $\mathrm{Dk}$ erhaltene Dotterkugeln im Keimwall, identisch mit denen des gelben Dotters. Winkel II/8.

Fig. 11. Mesoblastbildung in der area opaca, auf der innersten Zone des Keimwalls, ohne Verbindung mit dem Mesoblasten der area pellucida. A $\mathrm{p}$ area pellucida, A o area opaca. Epb, Msb, Hpb, die $3 \mathrm{Keim}$ biätter der area pellucida; nur das obere geht auf die area opaca über. HpblI verdicktes Ende des Hypoblasten an der inneren Grenze des Keimwalls. Kw Innerstes Stück des Keimwalls, bestehend aus Dotterkugeln mit spärlichen Zellen. A, B, C, verdickte Stellen des Mesoblasten der area opaca, noch ohne Verbindung untereinander. Winkel $\Pi / 8$.

Fig. 12. Spaltung des Mesoblasten der area opaca in drei Platten; erste Gefässbildung; Durchschnitt einer Substanzinsel. Gefüllte und leere Gefässe. Ep b Epiblast, Hf $p$ Hautfaserplatte, D f p Darmfaserplatte, G fp Gefässplatte des Mesoblasten. a I, a I, a III verdickte Stellen der Darmfaserplatte, entstanden durch Verwachsung der Gefässplatte mit ersterer. Dieselben sind Durchschnitte der in Fig. 16 und 19 gezeichneten Zellenringe um die Substanzinseln. G leeres Blutgefäss, G I, G I Hälften von gefüllten Gefässen. Die Gefässwand wird von der Darmfaserplatte und der Gefässplatte gebildet. Bl Blut. Winkel $11 / 8$.

Fig. 13. Innere Gränze des Keimwalls, Verhältniss der Zellen desselben zu den Dotterkugeln. Der Schnitt ist vor dem Kopfende des Embryo geführt. Die Dotterkugeln sind durch die Erhärtung in verschiedener Weise verändert worden. Epb Epiblast, $\mathrm{Dk}$ I leere Dotterkugeln, Dk II zum Theil gefüllte Dotterelemente, D k III Bruchstücke von Rindenschichten derselben, in situ verblieben. Der Keim ist 34 Stunden bebrütet. Kw Keimwall. Die Zellen liegen zwischen den Dotterkugeln. Winkel II 8 .

Fig. 14. Wachsthum des Mesoblasten in der area opaca durch Apposition von Keimwallzellen, der Mesoblast unregelmässig dick. Der Epiblast ist weggelassen, der Keimwall nur zum Theil ansgeführt. Ms b Mesoblast; bei a und b. Zellen, die sich aus dem Keimwall her an 
Die Entstehung des Blutes und der ersten Gefässe im Hühnerei. 595

die untere Fläche des Mesoblasten anlegen. Z Keimwallzellen. Winkel II/8.

Fig. 15. Concentrirung der Keimwallzellen zu Zellhaufen, und beginnende Wanderung dieser nach oben. Der Epiblast ist weggelassen. Ms b Mesoblast. a Zellhaufen im Mesoblasten, in eine Vertiefung des Keimwalls eingreifend, b Zellhaufen im Keimwall, durch einige Zellen mit dem Mesoblasten verbunden. Winkel II/8.

Fig. 16. Substanzinseln eines Keims von 11 Urwirbeln, von der Fläche gesehen (vgl. Fig. 12). Si Substanzinseln, A zellige Wand, B Inhalt derselben (Dotter), B I Blutbahn. Winkel II/7.

Fig. 17. Randvene eines Keims von 44 Stunden im Querschnitte. Spaltung des Mesoblasten in 3 Platten. Art der Gefässbildung wie in Fig. 12. Der Keimwall ist weggelassen. Epb Epiblast, $\mathrm{Hfp}$ Hautfaserplatte, gegen den Rand sich auflockernd. Hfp I Zellen, die zn ihr gehören, Dfp Darmfaserplatte, Gfp Gefässplatte. Winkel III/8.

Fig. 18. Keim von 11 Urwirbeln, Osmiumpräparat. Die eine Hälfte des hinteren Körperabschnitts ist gezeichnet. Ao innerer Theil der area opaca - area vasculosa, Ap area pellucida, Uw Urwirbel, Si ringförmige Substanzinseln, bei dieser Vergrösserung nur in der area pellucida deutlich. B1 Blutmassen, auf die area opa beschränkt. Winkel $\mathrm{II} / 1$.

Fig. 19. Vorderer Abschnitt von Fig. 18 bei stärkerer Vergrösserung. Bezeichnungen dieselben. Die ringförmigen Verwachsungsstellen zwischen Gefässplatte und Darmfaserplatte (Substanzinseln) sind leer gelassen, die Blutbahnen gelb gehalten. Unabhängiges Vorschreiten der Gefässe in die area pellucida. Winkel II/5.

Fig. 20. Flächenschnitt eines Keimwalls von 54 Stunden. Zellen in den Interstitien der Dotterkugeln liegend; die letzteren ganz oder zum Theil entleert. $\mathrm{Z}$ Keimwallzellen, $\mathrm{Dk}$ zum Theil gefüllte, $\mathrm{Dk}$ I leere Dotkugeln. Vgl. Fig. 13. Leitz I/7.

Fig. 21. Isolirte Keimwallzellen. $a$ und $b$ aus einem Keim von 11 Stunden, nach 4 wöchentlicher Maceration in diluirtem Alcohol, $c$ aus einem Keim von 16 Stunden nach 10 tägiger Maceration in $10 \%$ Kochsalzlösung. c I Zelle in Theilung. Vgl. Fig. ${ }^{* 6,7,9 . ~ L e i t z ~ I / 7 . ~}$

Fig. 22. Dotterformen aus dem gelben Dotter eines sehr früh während der Bebrütung abgestorbenen Ei's, ohne jeden Zusatz untersucht und gezeichnet. a aus Segmenten zusammengesetzte mehrkörnige Dotterkugel; b weiter fortgeschrittener Zerfall des Inhalts, aber im mittleren Segment weniger als in den beiden andern; C, $\mathrm{C}_{\mathrm{I}}$ feingranulirte gelbe Dotterkugeln; bei $d$ eine aus zahlreichen Segmenten bestehende gelbe Dottermasse; $d_{I}, d_{I n}, d_{\text {III }}$ grössere durchscheinende Inbalt3körner, an Kerne in feingranulirtem Protoplasma exinnernd, sie sind identisch mit den kleineren Körnern von a. Leitz I/7. 
596 J. Di isse: Die Entstehung der Blutes und der ersten Gefässe im Hübnerei.

Fig. 23. Aeusserster Rand der distalen. Zone eines macerirten und zerzupften Keimwalls von 11 Stunden. Form der Keimwallzellen deutlich erkennbar; D Fragmente von Dotterkugeln. Winkel II/8.

\section{Literatur.}

1) Remak, Untersuchungen über die Entwicklung der Wirbelthiere. Berlin, 1855.

2) His, Untersuchungen über die erste Anlage des Wirbelthierleibes. Leipzig, 1868.

3) His, Der Keimwall des Hühnereis und die Entstehung der parablastischen Zellen. Zeitschr. f. Anatomie und Entwicklungsgeschichte, Bd. I. pag. 274.1875.

4) Afanasieff, Ueber die Entwicklung der ersten Blutbahnen im Hühnerembryo. Wiener Sitzungsberichte Bd. 53. 1866.

5) Klein, Das mittlere Keimblatt in seinen Beziehungen zur Entwicklung der ersten Blutgefässe und Blutkörperchen. Wiener Sitzungsberichte Bd. 63. 1871.

6) Balfour, The development of the bloodvessels of the chick. Quarterly Journal of mikroskopical Science. 1873.

7) A. Goette, Ueber die Bildung der Keimblätter und des Blutes im Hühnerei. Archiv f. mikrosk. Anatomie X, 1874.

8) H. Virchow, Ueber das Epithel des Dottersacks im Hühnerei. Berliner Dissertation. 1875.

9) Kölliker, Entwicklungsgeschichte, 2. Aufl. Leipzig 1876.

10) Disse, Die Entwicklung des mittleren Keimblatts im Hühnerei. Archiv f. mikroskop. Anatomie. Bd. XV. 1878. 
Fig.16.

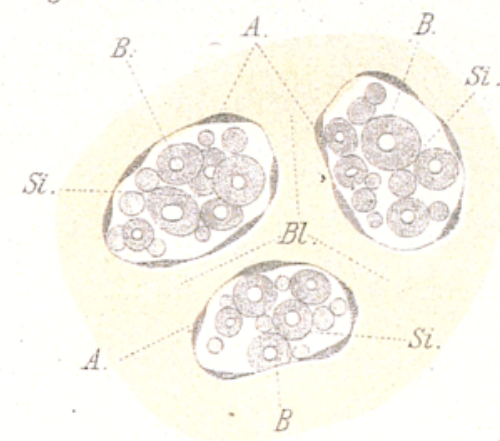

Fig.12.
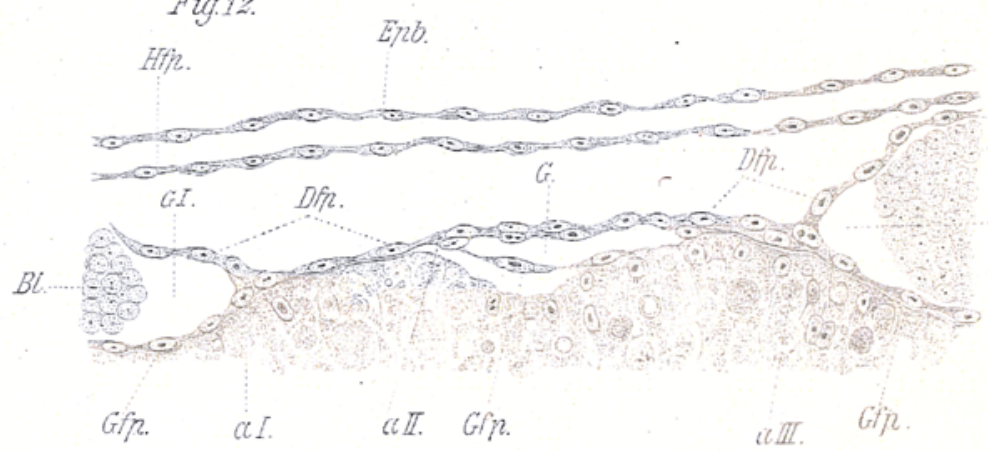

Bl. GII.
Gfp.
$a 1$
aII. Gfr.

Fig. 17.

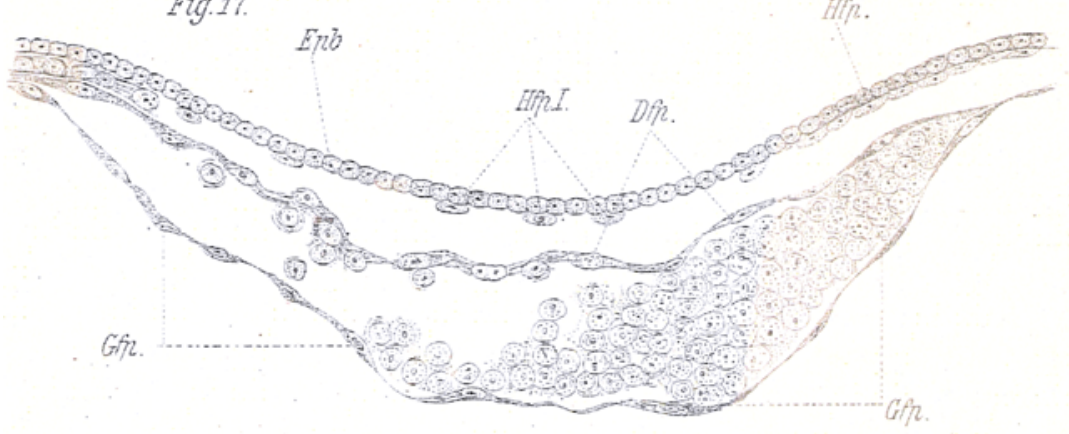

Fig. 23

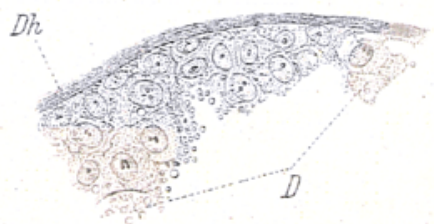

